OPEN ACCESS

Edited by:

Wei Zhao,

Chengdu Medical College, China

Reviewed by:

Jing Zhou,

Guangxi Medical University, China

Chuanbin Yang,

Jinan University, China

*Correspondence:

Xiang-Dong Cheng

chengxd516@126.com

Jiang-Jiang Qin

zylysjtu@hotmail.com

${ }^{\dagger}$ These authors have contributed equally to this work

Specialty section:

This article was submitted to Molecular and Cellular Oncology, a section of the journal Frontiers in Cell and Developmental

Biology

Received: 26 October 2020 Accepted: 12 November 2020 Published: 03 December 2020

Citation:

Xu J-L, Yuan L, Tang Y-C, Xu Z-Y,

$X u H-D$, Cheng $X-D$ and Qin J-J

(2020) The Role of Autophagy in Gastric Cancer Chemoresistance:

Friend or Foe?

Front. Cell Dev. Biol. 8:621428 doi: 10.3389/fcell.2020.621428

\section{The Role of Autophagy in Gastric Cancer Chemoresistance: Friend or Foe?}

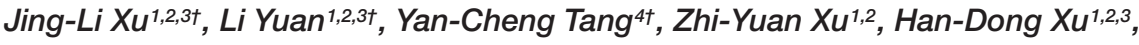 \\ Xiang-Dong Cheng ${ }^{1,2 *}$ and Jiang-Jiang Qin ${ }^{1,2 *}$
}

${ }^{1}$ Institute of Cancer and Basic Medicine, Chinese Academy of Sciences, Hangzhou, China, ${ }^{2}$ Cancer Hospital of the University of Chinese Academy of Sciences, Zhejiang Cancer Hospital, Hangzhou, China, ${ }^{3}$ The First Clinical Medical College of Zhejiang Chinese Medical University, Hangzhou, China, ${ }^{4}$ School of Chinese Medicine, Hong Kong Baptist University, Kowloon Tsai, Hong Kong, China

Gastric cancer is the third most common cause of cancer-related death worldwide. Drug resistance is the main inevitable and vital factor leading to a low 5-year survival rate for patients with gastric cancer. Autophagy, as a highly conserved homeostatic pathway, is mainly regulated by different proteins and non-coding RNAs (ncRNAs) and plays dual roles in drug resistance of gastric cancer. Thus, targeting key regulatory nodes in the process of autophagy by small molecule inhibitors or activators has become one of the most promising strategies for the treatment of gastric cancer in recent years. In this review, we provide a systematic summary focusing on the relationship between autophagy and chemotherapy resistance in gastric cancer. We comprehensively discuss the roles and molecular mechanisms of multiple proteins and the emerging ncRNAs including miRNAs and IncRNAs in the regulation of autophagy pathways and gastric cancer chemoresistance. We also summarize the regulatory effects of autophagy inhibitor and activators on gastric cancer chemoresistance. Understanding the vital roles of autophagy in gastric cancer chemoresistance will provide novel opportunities to develop promising therapeutic strategies for gastric cancer.

Keywords: gastric cancer, autophagy, chemoresistance, ncRNAs, natural products, inhibitor and activator

\section{INTRODUCTION}

Gastric cancer is one of the most common gastrointestinal tumors in the world, with more than one million new cases every year, and remains the third leading cause of cancer-related deaths (Fitzmaurice et al., 2019; Thrift and El-Serag, 2019). For patients with early gastric cancer, surgical resection is the best treatment option. However, more than $60 \%$ of patients have developed local or distant metastasis at diagnosis, which causes that most of the patients do not have the opportunity to receive surgical treatment (Yuan et al., 2020). Hence, chemotherapy-based comprehensive treatment is the main choice for most patients with middle- and late-stage gastric cancer (Zhang et al., 2020). However, poor or even no response to chemotherapy is frequently observed in the treatment of gastric cancer patients due to the intrinsic or acquired drug resistance, which becomes the most detrimental cause of treatment failure and low survival rate (Biagioni et al., 2019).

Autophagy, as a major intracellular degradation system in eukaryotic cells that degrades and clears defective or aging organelles, can be divided into three major forms according to the 
different mechanisms of clearing intracellular components: macroautophagy, microautophagy, and chaperone-mediated autophagy (Galluzzi and Green, 2019). Among them, macroautophagy (hereafter autophagy) is the most common and intensively studied form (Amaravadi et al., 2019; Abdrakhmanov et al., 2020). Previous studies have shown that autophagy plays a double-edged sword role at different stages of tumorigenesis. At the early stage, autophagy plays a "tumor-suppressor" function, which can stabilize the genome, protect the damaged tissues and cells, and suppress tumor occurrence, proliferation, invasion, and metastasis (Høyer-Hansen and Jäättelä, 2008). On the contrary, once the tumor is formed, autophagy plays an oncogenic function that provides cancer cells with needed survival contexts, such as nutrition to resist stress (especially after chemotherapy treatment) (Kimmelman and White, 2017). The specific roles of autophagy in tumors depend on tumor type and tumor heterogeneity, which is regulated by multiple proteins and non-coding RNAs (ncRNAs) (Jiang et al., 2020; Perez-Montoyo, 2020).

Traditional medicine (TM) has been widely used in China, South Korea, and Japan for thousands of years to treat various diseases, usually in the form of decoctions with 2-15 kinds of herbs (Qin et al., 2017; Hempen and Hummelsberger, 2020). Numerous TM decoctions or natural products derived from medical herbs have been proven to have anticancer activities (Li et al., 2013; Qin et al., 2013; Yang et al., 2019; Yuan et al., 2019). Also, dietary natural products have been shown to regulate autophagy and thus enhance the chemosensitivity of cancer cells (Emanuele et al., 2018; Dutta et al., 2019). Some antibiotics, antiinflammatory drugs, anesthetics, etc. have been shown to regulate autophagy to exert anticancer effects (Zhu et al., 2013; Jiang et al., 2019; Moon et al., 2019; Petroni et al., 2020).

The roles of autophagy in gastric cancer progression, metastasis, and prognosis have been extensively discussed in several recent reviews (Qian and Yang, 2016; Cao et al., 2019; Zhang F. et al., 2019). Herein, we provide a review focusing on the relationship between autophagy and chemotherapy resistance in gastric cancer. We comprehensively discuss the roles and molecular mechanisms of multiple proteins and newly identified ncRNAs including miRNAs and long non-coding RNAs (lncRNAs) in autophagy and gastric cancer chemotherapy resistance, as well as their potential as therapeutic targets for gastric cancer precise medicine. We also summarize the natural products and other drugs that exhibit regulatory effects on autophagy and chemotherapy resistance. Summarizing the process of autophagy in detail and classifying the different roles of autophagy in gastric chemoresistance will provide a comprehensive understanding of autophagy and develop promising therapeutic strategies for gastric cancer.

\section{OVERVIEW OF AUTOPHAGY}

Autophagy is a common, complex, physiological and pathological, and highly conserved self-metabolic process (Galluzzi and Green, 2019). During certain extreme conditions like starvation, hypoxia, or other environmental stresses, autophagy is also an indispensable process to provide energy for cell regeneration and cell survival, which functions as a cytoprotective mechanism. However, autophagic cell death, also called type II-programmed cell death, may be accompanied by excessive autophagy (Qi et al., 2020). Here, we summarize the latest updates on the process and related signaling pathways of autophagy.

\section{Process of Autophagy}

The process of autophagy can be divided into the following stages (Figure 1): (1) initiation, (2) vesicle nucleation, (3) vesicle elongation and maturation, (4) vesicle fusion, and (5) cargo degradation (Levy et al., 2017). Each stage of autophagy is regulated by a variety of autophagy-related genes (ATGs) such as ATG5, ATG7, ATG12, ATG16L1, and their complexes, etc. (Levine and Kroemer, 2019).

\section{Initiation and Nucleation}

Upon nutrient deficiency, hypoxia, and inflammation, autophagy is initiated by the unc-51-like kinase 1 (ULK1) complex (Figure 2A), composing of ULK1, FIP200 (FAK family kinaseinteracting protein of $200 \mathrm{kDa}$, also known as ATG17), ATG13, and ATG101. Specifically, the intrinsically disordered region (IDR) of ATG13 interacts with ULK1 and FIP200 to form a pre-autophagosomal structure (PAS) (Hollenstein and Kraft, 2020), while its HORMA (Hop1, Rev7, and MAD2) domain forms a dimer with ATG101's HORMA domain (Kim B. W. et al., 2018). In addition, the N-terminal 640 residues (NTD) of FIP200, shaping like a letter C with the presence of ATG13, has an intimate interaction with C-terminal IDR of ATG13 and C-terminal early autophagy targeting/tethering (EAT) domain of ULK1, ensuring the successful initiation of autophagy (Shi et al., 2020). Following the activation of ULK1 complex, the class III phosphatidylinositol 3-kinase (PI3KC3) complex, which consists of vacuolar protein sorting 34 (VPS34, also known as PI3KC3), ATG14, the activating molecule in BECN1-regulated autophagy protein 1 (AMBRA1), and the scaffold protein Beclin-1, generates phosphatidylinositol 3-phosphate (PI3P) at an endoplasmic reticulum (ER) subdomain named omegasome (Young et al., 2019; Sanchez-Martin et al., 2020). Subsequently, PI3P recruits certain effector proteins, including WIPIs (WD repeat domain phosphoinositide-interacting proteins) (Figure 2B), which can bind ATG16L1 (Bakula et al., 2017).

\section{Elongation and Maturation}

ATG5-ATG12 complex interacts with ATG16L1 to form the ATG12-ATG5-ATG16L1 complex, the crucial element for autophagosome elongation (Lystad et al., 2019). ATG12-ATG5ATG16L1 complex cooperating with ATG7 and ATG3 recruits LC3 (microtubule-associated protein 1 light chain 3 alpha) to lipid phosphatidylethanolamine (PE) (Lystad et al., 2019). This type of LC3 is called LC3-II, which can elongate and close membranes in the outer surfaces of the autophagosome membrane and bind with cargo receptors such as p62 to select proteins or organelles (Zhang X. et al., 2019). In addition, recent studies have highlighted that TRAPP III-specific proteins (TRAPPC11) are necessary for the close of isolation membranes 


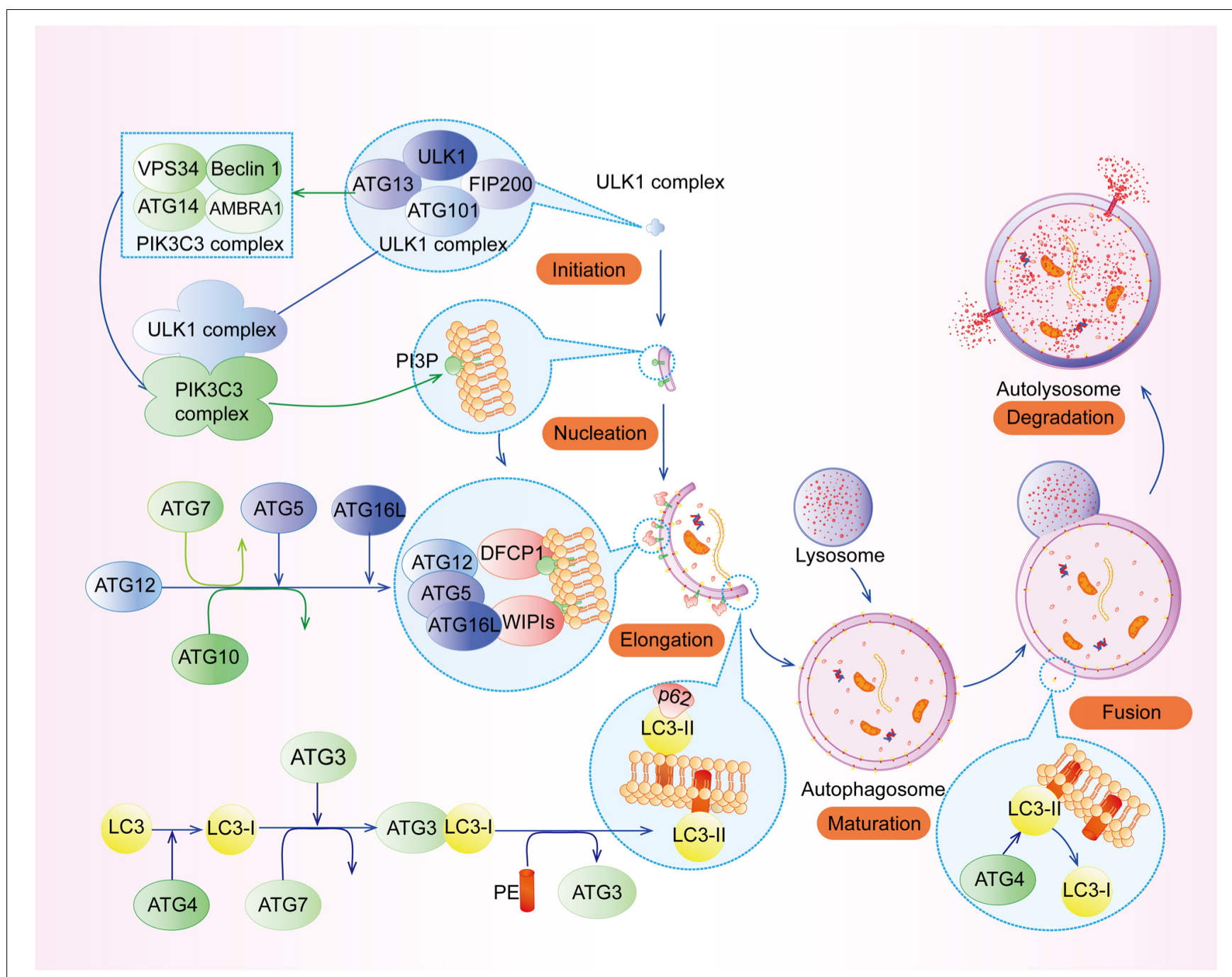

FIGURE 1 | The process of autophagy. The process of autophagy can be divided into the following stages: (1) initiation, (2) vesicle nucleation, (3) vesicle elongation and maturation, (4) vesicle fusion, and (5) cargo degradation.

and recruitment of WIPI4-ATG2 to isolation membranes with the presence of ATG9, perhaps due to its carboxy-terminus, while TRAPPC12 is downstream of TRAPPC11 (Stanga et al., 2019). Interestingly, Guardia et al. (2020) have found that ATG9A (human ATG9), a homotrimer with four transmembrane helices, interacts with ATG2 at C-terminal platform domain of ATG9A (1-723 construct and 1-522 construct) to transfer lipids from ATG2 to ATG9A, and then ATG9A can induce the autophagosome membrane curvature for the further processes of autophagy.

\section{Fusion and Degradation}

After the formation and maturation of the autophagosome, most of the proteins in the outer membrane are dissociated, including ATG proteins. Then, the autophagosome fuses with the lysosome (Figure 2C) to form autolysosome in the perinuclear region (Lőrincz and Juhász, 2020). In this special area, SNAREs (soluble $\mathrm{N}$-ethylmaleimide-sensitive factor attachment protein receptors), Syntaxin17, and SNAP29 located at autolysosome and VAMP8 located at lysosome play indispensable roles in this process (Diao et al., 2015). The localization of Syntaxin17SNAP29 complex is regulated by vesicle-associated membrane protein 2 (LAMP2), while localization of Syntaxin17 is also regulated by LC3 due to its LC3-interacting region (LIR) (Corona and Jackson, 2018). However, recent research has found that LAMP2 can regulate this fusion without the function of Syntaxin17 in human cardiomyocytes (Chi et al., 2019). There are many other regulators of autolysosome fusion, including RAB7, NRBF2, PLEKHM1, TECPR1, RUFY4, and so on (Reggiori and Ungermann, 2017; Cai et al., 2020). At the same time, LC3-II located on outer surfaces of the membrane can be disconnected from PE by ATG4 for recycling, but internal LC3-II is degraded with cargoes and p62 by lysosomal enzymes (Nakayama et al., 2017). Finally, products of decomposition can be recycled and provided for cell growth. 

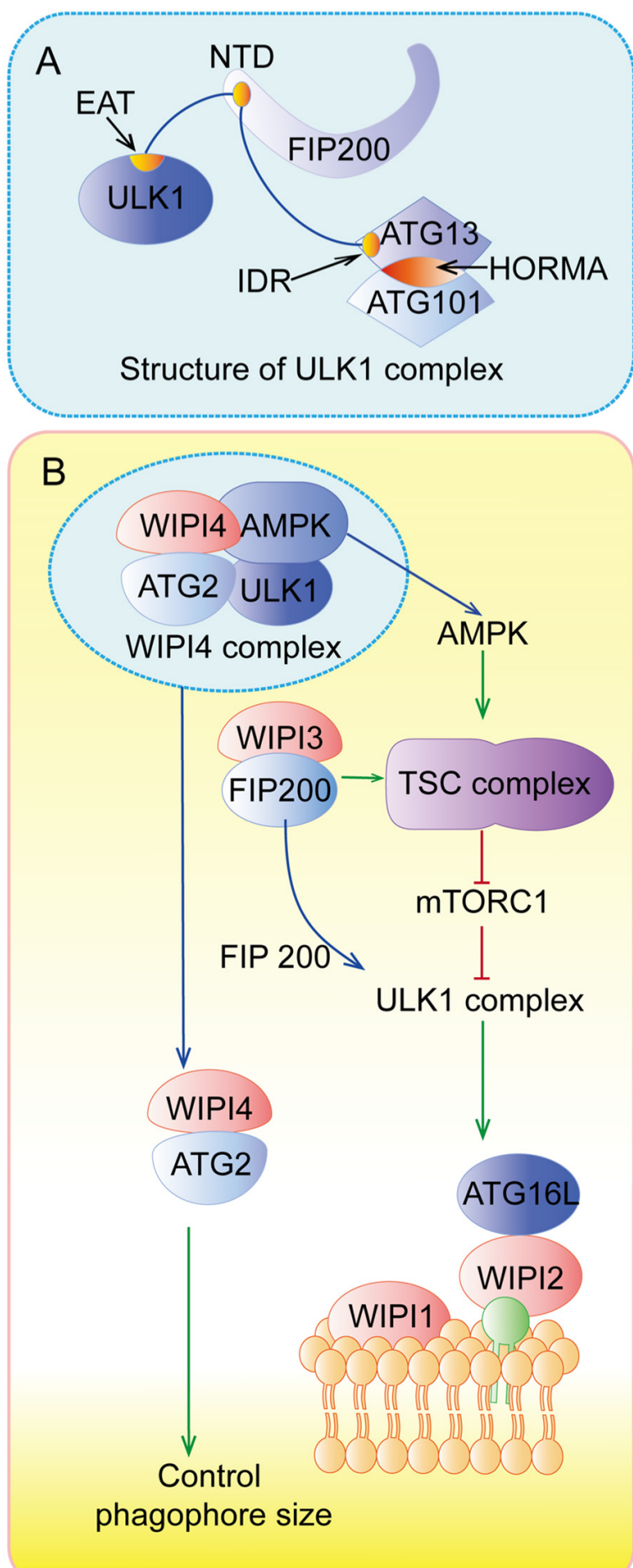

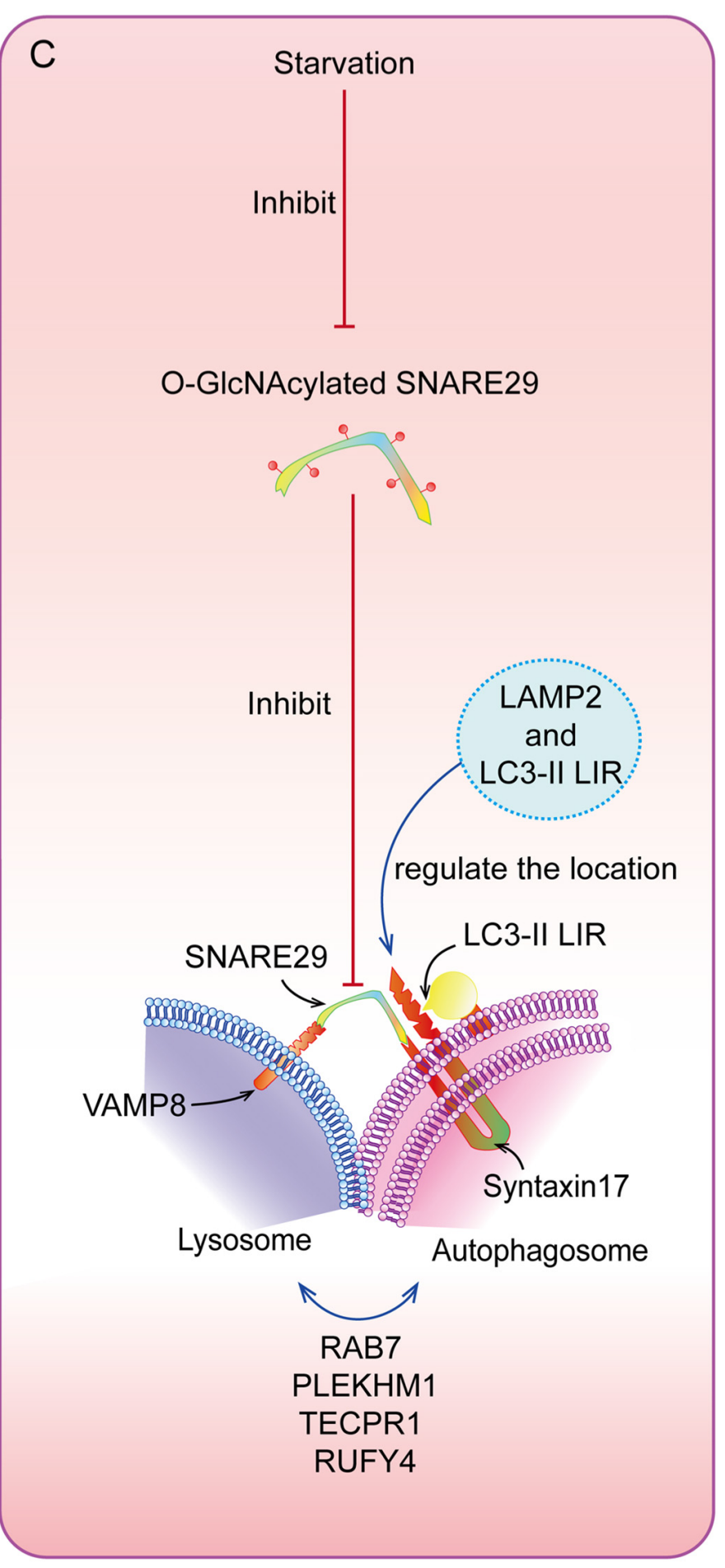

FIGURE 2 I Special parts of autophagy. (A) The structure of ULK1 complex. (B) The functions of WIPIs in autophagy. (C) The fusion between lysosome and autolysosome.

\section{Signal Pathways Regulating Autophagy}

Mammalian target of rapamycin (mTOR) complex 1 (mTORC1, made up of mTOR, Raptor, Deptor, mLST8, and PRAS40) and mTOR complex 2 (mTORC2, composed of mTOR, Rictor,
Deptor, mLST8, Sin1, and PRA5/Protor-1) have a common central kinase, mTOR, a conserved serine/threonine protein kinase, which plays important roles in multiple biological processes involving in cell growth, such as regulating autophagy 
(Murugan, 2019). Previous studies have shown that multiple signaling pathways, such as PI3K/AKT, MAPK (mitogen activated kinase-like protein), AMPK (protein kinase AMPactivated catalytic subunit alpha 1 , also known as PRKAA1) (Figure 3), p53, and PTEN (phosphatase and tensin homolog) pathways, can regulate mTORC1 and autophagy (Figure 4).

\section{PI3K/AKT Pathway}

The pro-oncogenic class-I PI3K (PI3KC1) can catalyze the phosphorylation of phosphatidylinositol-4,5-bisphosphate (PI4,5-P2) into phosphatidylinositol-3,4,5-trisphosphate (PIP3), which binds both AKT and phosphoinositide-dependent protein kinase 1 (PDK1), resulting in the AKT phosphorylation at Thr308 by PDK1 (Parikh et al., 2012). The complete activation of AKT, in turn, activates its downstream mTORC1, contributing to the suppression of autophagy (Figure 3). In addition, phosphorylated AKT can phosphorylate the Ser939 and Thr1462 residues of TSC2 to block the formation of TSC1 (TSC complex subunit 1)/TSC2 complex, an inhibitor of Ras homolog enriched in the brain (Rheb). TSC1/TSC2 complex acts as a guanosine triphosphatase (GTPase)-activating protein (GAP), which can promote RhebGTP into Rheb-GDP; the former can activate mTORC1 while the latter cannot (Fawal et al., 2015; Yu et al., 2017). Besides, AKT can also be phosphorylated by mTORC2 at Ser473 and then the activated AKT promotes its downstream regulator, causing the inhibition of autophagy (Li et al., 2018).

\section{MAPK Pathway}

MAPKs play indispensable roles in many cellular processes, including cell growth, proliferation as well as autophagy (Anerillas et al., 2020). There are many subtypes of MAPKs whose upstream regulators are different, including mitogenactivated protein 3 kinases (MAPKKKs) and mitogen-activated protein 2 kinases (MAPKKs) (Gomez-Osuna et al., 2020). c-Jun N-terminal kinase (JNK) and extracellular signal-regulated kinase (ERK) are two classical and important types of MAPKs (Anerillas et al., 2020). Under various stresses (Figure 3), JNK can phosphorylate and activate BCL-2, leading to the dissociation of Beclin-1, a crucial member of PI3KC3, from BCL-2 and the subsequent induction of autophagy (Fan et al., 2016; Cheng et al., 2019). On one hand, the activated ERK1 and ERK2 can induce autophagy through Ras-Raf (MAPKKK)-MEK (MAPKK)-ERK signal pathway (Panda et al., 2015). On the other hand, the phosphorylated ERK1/2 can block the TSC1/TSC2 complex, leading to the increased expression of Rheb-GTPase to induce mTORC1, which can result in the inhibition of autophagy (Pattingre et al., 2003; Ranek et al., 2019).

\section{AMPK Pathway}

AMPK pathway is a key pathway to preserve energy balance and coordinate metabolism in eukaryotic cells, which is extensively involved in the regulation of autophagy, apoptosis, epithelialmesenchymal transition (EMT), etc. (Carling, 2017; Zhang et al., 2018). Under the depletion of nutrients and energy with the increasing AMP/ATP ratio, LKB1 (liver kinase B1), a tumor suppressor kinase, stimulates the activation of AMPK, which subsequently promotes the formation of TSC1/TSC2 complex to inactivate mTORC1 and induce autophagy (Figure 3) (Bakula et al., 2017). Moreover, AMPK can inhibit mTORC1 by directly phosphorylating Raptor (Barroso-Chinea et al., 2020). Under the undernourished condition, AMPK can directly phosphorylate ULK1 at Ser317, S467, S555, S574, S637, and Ser777 by separating mTORC1 from ULK1 and stimulate autophagy (Zachari and Ganley, 2017), whereas mTORC1 can block the function of AMPK by phosphorylating ULK1 under the glucose abundant condition (Mao and Klionsky, 2011).

\section{P53 Pathway}

The p53 tumor suppressor participates in multiple cellular biological processes, including cell cycle arrest, apoptosis, senescence, proliferation, DNA repair, and autophagy (Qin et al., 2018; Hafner et al., 2019). However, the mechanisms of nuclear p53 and cytoplasmic p53 are different. With the expression of nuclear p53 stimulated by starvation conditions, the activated AMPK induces autophagy through the TSC1/TSC2 complex and mTOR pathway (Figure 4) (Hu et al., 2019; Gao et al., 2020). Chollat-Namy et al. (2019) have found that p53 upregulates the expression of its downstream targets Sestrin-1 and Sestrin-2, which further activate AMPK through phosphorylating AMPK at Thr172, subsequently leading to the dephosphorylation of mTORC1 at S2448 and the induction of autophagy. Damageregulated autophagy modulator (DRAM) and death-associated protein kinase 1 (DAPK1) are other important downstream targets of p53 and have been reported to induce autophagy (Crighton et al., 2006; Zhou et al., 2016; Hu et al., 2019). At the same time, the activated DAPK1 can, in turn, stimulate and stabilize p53, forming a positive feedback loop (Hu et al., 2019). In contrast, cytoplasmic p53 inhibits autophagy through blocking AMPK, TIGAR (TP53-induced glycolysis and apoptosis regulator), and Beclin-1, whose underlying mechanisms need to be further studied (Mrakovcic and Fröhlich, 2018).

\section{PTEN Pathway}

The tumor suppressor PTEN has been found to act as a negative endogenous regulator of the PI3K/AKT pathway, which can hydrolyze PIP3 back to PIP2, resulting in the induction of autophagy (Figure 4) (Sun et al., 1999; Gao et al., 2018). It has also been reported that PTEN can play the role of autophagy catalysator through the AKT/mTOR pathway (Chen et al., 2015; Gao et al., 2018). PTEN regulates autophagy in PTEN/AKT/FOXO3a/ATG7 axis in non-small-cell lung cancer (NSCLC) cells, independent of mTOR and Beclin-1 (Cai et al., 2018). Specifically, the overexpression of PTEN inhibits AKT activity, which leads to the increased ATG7 expression through FOXO3a (Cai et al., 2018). Chen et al. (2015) have found that the phosphorylation of PTEN at Ser113 makes PTEN located in the nucleus, resulting in the activation of the MAPK8/JNK1MAPK9/JNK2-sestrin2-AMPK pathway to facilitate autophagy. Furthermore, upon mitochondria damage, the accumulated PINK1 (PTEN induced putative kinase 1) can induce autophagy by blocking the release of cytochrome $c$, which needs further investigations (Boosani et al., 2019). 
(2)

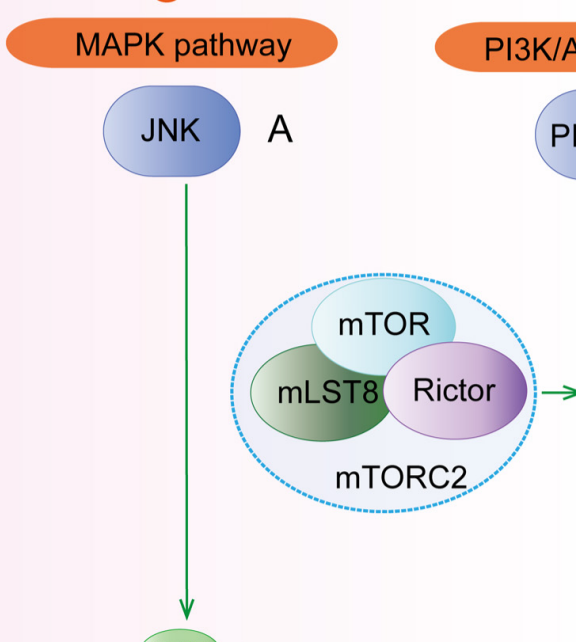

(1)

$\mathrm{KT} / \mathrm{mTOR}$

$\mathrm{S} 70^{\mathrm{S} 87} \mathrm{T69}$

BCL-2

BCL-2

Beclin 1

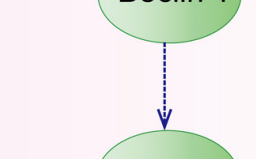

Beclin 1
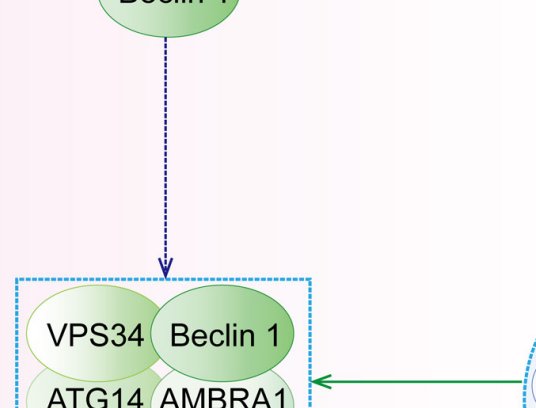

ATG14 AMBRA1

PIK3C3 complex<smiles></smiles>
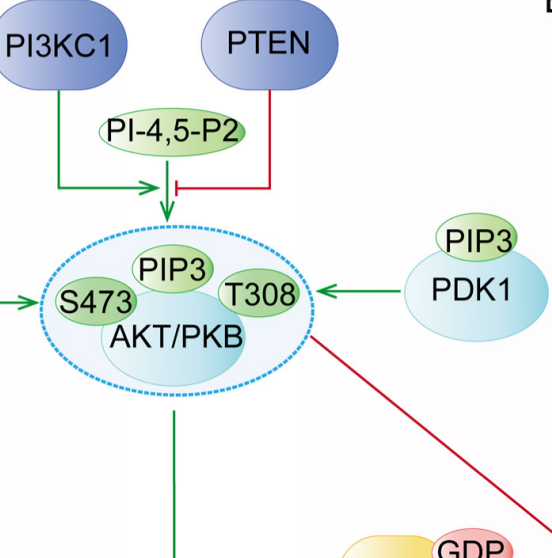

$b^{G}$

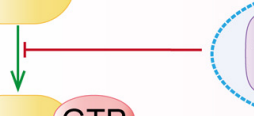

TSC1 TSC2

Rheb GTP

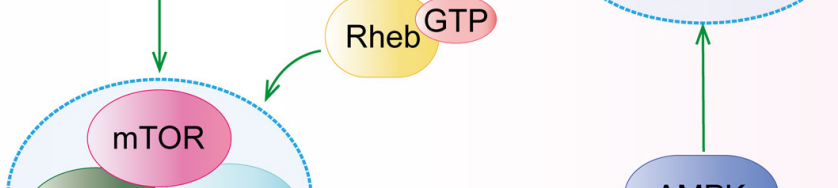

AMPK
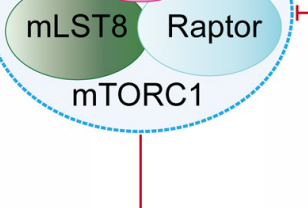$$
37
$$
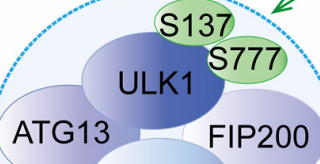

FIP200

ATPIAMP $\downarrow$

AMPK pathway

(3)

FIGURE 3 | The major signaling pathways involved in the regulation of autophagy. (1) PI3K/AKT pathway: under stresses, the activated PI3KC1 makes PI-4,5-P2 into PIP3 and then activates AKT with the help of PDK1. The activated AKT, directly and indirectly, activates mTORC1 (composed of mTOR, mLST8, and Raptor) to inhibit ULK1 complex and autophagy. Specifically, the indirect pathway includes the TSC1/TSC2 complex and Rheb-GTP. (2) MAPK pathway: the major regulators include the JNK pathway and the ERK pathway. (A) JNK can separate the conjunction of Beclin-1 and BCL-2 by phosphorylating BCL-2 at Thr69, Ser70, and Ser87, and then Beclin-1 participates in the assembly of PIK3C3 complex to induce autophagy. (B) ERK has dual roles in autophagy. The successively activated Ras-Raf-MEK-ERK1/2 can directly induce autophagy. Besides, the activated ERK1/2 can block the TSC1/TSC2 complex and regulate its downstream to inhibit autophagy. (3) AMPK pathway: Under some conditions with the increasing AMP/ATP ratio, the activated AMPK promotes the formation of the TSC1/TSC2 complex to inactivate mTORC1 and regulates its downstream regulators. AMPK can also phosphorylate ULK1 at Ser317, Ser777, S467, S555, S574, and S637 to stimulate autophagy. 
(1)

\section{p53 pathway}

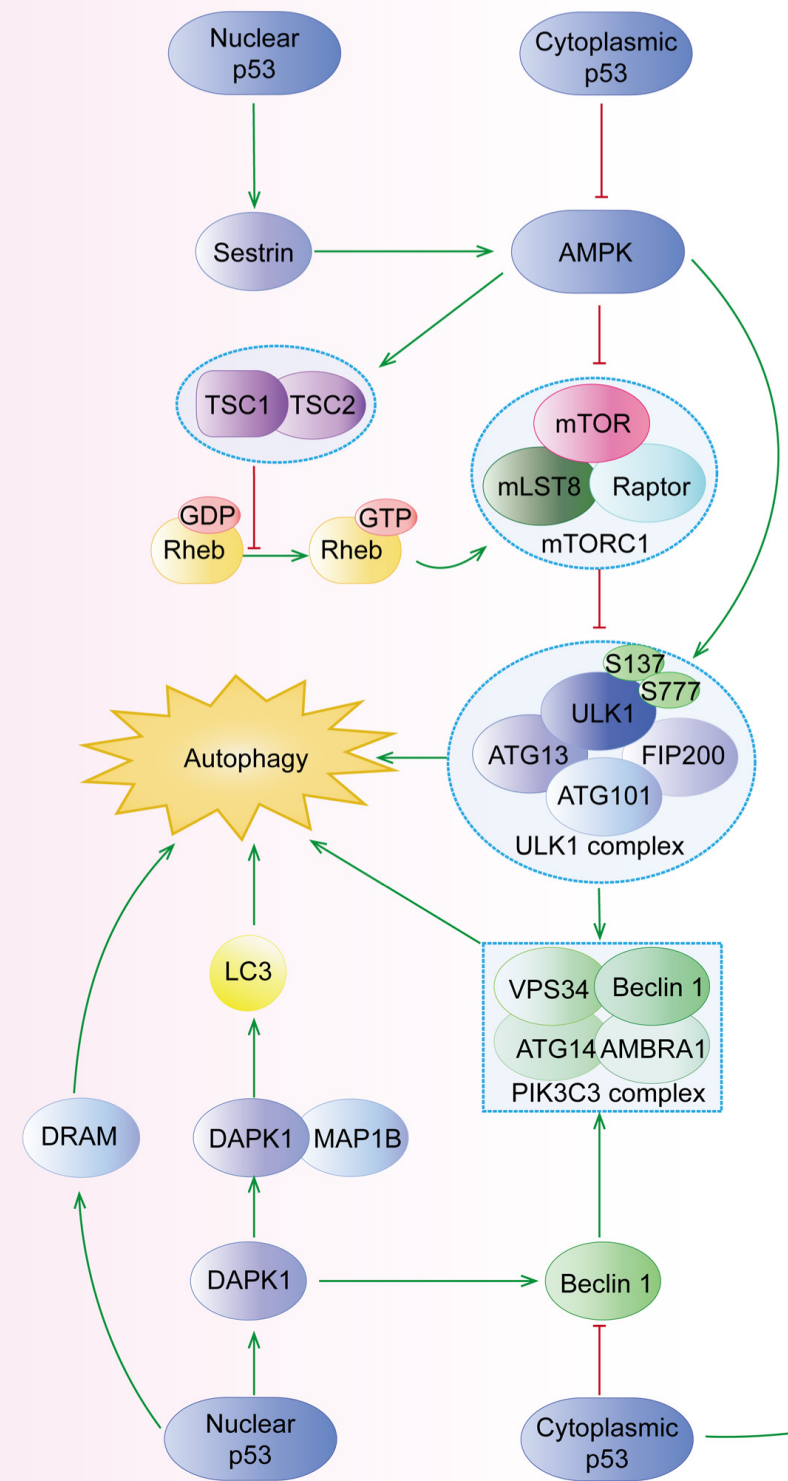

(2)

PTEN pathway 
cancer chemoresistance (Tsai et al., 2020; Zeng et al., 2020). Therefore, if properly applied, targeting autophagy may be an important strategy for the prevention and treatment of chemoresistance. In this section, we provide a summary of the regulation of autophagy by various proteins (Table 1) and ncRNAs (Table 2) as well as its different roles in gastric cancer chemoresistance (Figure 5).

\section{Key Regulatory Proteins in Autophagy-Mediated Gastric Cancer Chemoresistance}

Several proteins have shown regulatory effects on autophagymediated gastric cancer chemoresistance (Figure 5A) by (i) inducing both autophagy and chemoresistance; (ii) inhibiting autophagy but inducing chemoresistance; or (iii) inhibiting both autophagy and chemoresistance. Based on the modulatory effects of these proteins on autophagy and chemoresistance, developing their inhibitors or activators and combining these compounds with autophagy inhibitors or activators have been emerging as a promising strategy to overcome chemotherapy resistance.

\section{Regulatory Proteins Inducing Both Autophagy and Chemoresistance}

It has recently been reported that several proteins (Table 1) can induce autophagy by regulating autophagy regulatory genes, such as Beclin-1, ATG12, ATG5, p62, LC3-I, LC3-II, and so on. Nie et al. (2020) have reported that wiskott-aldrich syndrome protein family member 3 (WASF3) is an obstacle for the sensitivity of gastric cancer to oxaliplatin (OXA). It has been found that WASF3 overexpression induces autophagy by increasing ATG12 level and causes OXA resistance in gastric cancer cells, while interference with WASF3 can reverse OXA resistance in vitro (Nie et al., 2020). Chen et al. (2019) have demonstrated that the expression of polo-like kinase 1 (PLK1) is significantly increased in cisplatin (DDP)-resistant SGC7901/DDP cells, whereas PLK1 knockdown inhibits autophagy, increases apoptosis, and restores the chemosensitivity of DDPresistant cells. Aquaporins (AQPs) are a family of small integral membrane proteins; among them, AQP3 is highly expressed in gastric cancer tissues (Moosavi and Elham, 2020). Dong et al. (2016) have shown that the upregulation of AQP3 increases the expression of ATG5 and Beclin-1, decreases the expression of p62, induces autophagy, and causes DDP resistance, while the application of autophagy inhibitor chloroquine (CQ) can reverse DDP resistance. DJ-1, also known as Parkinson's disease associated protein 7 (PARK7), is highly expressed in several types of cancer and has been proposed as a chemoresistance-related factor (Jin, 2020). Indeed, DJ-1 is highly expressed in gastric cancer tissues and Epirubicin (EPI)-resistant gastric cancer cells, and EPI treatment can increase the expression of DJ-1 in a dosedependent manner (Pan et al., 2018). Pan et al. (2018) have reported that the overexpression of DJ-1 significantly increases LC3-II level, induces autophagy, and attenuates EPI-induced apoptosis, while DJ-1 knockdown can reduce autophagy and increase apoptosis, thereby reversing EPI resistance in MGC803 and SGC7901 gastric cancer cells.
Some regulatory proteins have been found to induce autophagy and chemoresistance by regulating the $\mathrm{PI} 3 \mathrm{~K} / \mathrm{AKT} / \mathrm{mTOR}$ signaling pathway (Table 1). Tetraspanin 9 (TSPAN9), a member of four transmembrane protein superfamily that plays an important role in tumor progression, has shown regulatory effects on proliferation, migration, invasion, and autophagy (Qi et al., 2019, 2020). Qi et al. (2020) have reported that the protein level of TSPAN9 is increased in 5-fluorouracil (5-FU)-resistant gastric cancer cells, which inhibits the catalytic activity of PI3K through binding to PIK3R3 (p55) and promotes autophagy, whereas knockdown of p55 can enhance the sensitivity of gastric cancer cells to 5-FU. The cluster of differentiation 133 (CD133), an important stemness-related marker has also been related to autophagy (Liu Y. C. et al., 2020b). Lu et al. (2019) have reported that the expression level of CD133 and the percentage of CD133-positive cells are increased in DDP-resistant gastric cancer cells, promoting cancer stem cell (CSC) properties of DDP-resistant cells. Further studies have shown that CD133 can increase autophagy and the stemness of gastric cancer cells via activating the $\mathrm{PI} 3 \mathrm{~K} / \mathrm{AKT} / \mathrm{mTOR}$ signaling pathway, causing DDP resistance of gastric cancer cells in vivo and in vitro (Lu et al., 2019).

The regulatory proteins of the AMPK pathway have been shown to induce autophagy and chemoresistance (Table 1). Xiao et al. (2020) have suggested that tripartite motif containing 14 (TRIM14) is significantly up-regulated in 5-FU- and OXAresistant gastric cancer tissues and cell lines. Further studies have shown that TRIM14 can promote autophagy and induce chemoresistance by activating the AMPK/mTOR pathway in vivo and in vitro while silencing TRIM14 provides the opposite effects (Xiao et al., 2020). Pei et al. (2018) have found that metadherin $(\mathrm{MTDH})$ expression in gastric cancer tissues is significantly increased and positively correlated with 5-FU resistance. Further mechanism studies have suggested that MTDH can promote AMPK phosphorylation, upregulate ATG5 expression, activate autophagy, and consequently induce 5-FU resistance (Pei et al., 2018).

\section{Regulatory Proteins Inhibiting Autophagy but Inducing Chemoresistance}

Embryonic stem cell-expressed Ras (ERas) is a novel member of the Ras protein family and is highly expressed in gastric, colorectal, and breast cancer (Suárez-Cabrera et al., 2018; Tian et al., 2019). Tian et al. (2019) have found that ERas overexpression significantly increases the phosphorylation level of mTOR (Ser2448) and its substrate (ULK1-Ser757), as well as AKT-Ser473, activates the AKT/mTOR pathway, blocks autophagic flux in gastric cancer cells, suppresses DDPinduced apoptosis, and induces DDP resistance. In contrast, silencing ERas has been found to increase autophagic flux and enhance the sensitivity of gastric cancer cells to DDP in vitro (Tian et al., 2019).

Chemokine C-C motif ligand 2 (CCL2), is a well-known cytokine belonging to the CC chemokine family 9, which can affect drug sensitivity in both paracrine and autocrine manners (Hao et al., 2020). CCL2 expression has been related to tumor invasion, metastasis, and drug resistance (Zhong et al., 2018; 
Dutta et al., 2020; Hao et al., 2020). Xu et al. (2018b) have found that DPP-resistant gastric cancer cell lines, such as BGC823/DDP cells and SGC7901/DDP cells secrete more CCL2 to maintain DDP resistance. It has further been found that CCL2 overexpression increases the expression of p62 by activating $\mathrm{PI} 3 \mathrm{~K} / \mathrm{AKT} / \mathrm{mTOR}$ signaling pathway, whilst the increased expression of $\mathrm{p} 62$, in turn, activates the transcription of CCL2, inhibits autophagy, and forms a positive feedback loop to maintain drug resistance (Xu et al., 2018b).

Rab5a, also known as RAB5, is a member of the Rab family of small GTPases and is involved in the autophagy process by blocking autophagosome-lysosome fusion (Lu et al., 2014). $\mathrm{Xu}$ et al. (2018a) have shown that Rab5a expression level is positively associated with drug resistance in gastric cancer cells. It has been found that Rab5a overexpression increases the phosphorylation level of mTOR, decreases the LC3-II/I ratio, and increases p62 expression level, thereby inhibiting autophagy and inducing DDP resistance, whereas Rab5a knockdown can facilitate autophagy and reverse DDP resistance in vitro (Xu et al., 2018a).

\section{Regulatory Proteins Inhibiting Both Autophagy and Chemoresistance}

O-6-methylguanine-DNA methyltransferase (MGMT) is a type of suicide DNA damage repair enzymes, and DNA damage repair disorders have been reported to induce autophagy (Balvers et al., 2015; Abdu et al., 2020). Lei et al. (2020) have found that the high expression of MGMT is significantly correlated with the low expression of ATG4B and the favorable prognosis of gastric cancer. DDP can inhibit MGMT expression in a dose- and timedependent manner and the low expression of MGMT can, in turn, induce autophagy and cisplatin resistance. Overexpression of MGMT can inhibit autophagy and reverse DDP resistance in vivo and in vitro (Lei et al., 2020).

TABLE 1 | Key regulatory proteins in autophagy-mediated chemoresistance of gastric cancer.

\begin{tabular}{|c|c|c|c|c|c|c|}
\hline Protein & Expression in GC & Effects on autophagy & Chemotherapy & Effects on chemosensitivity & Downstream pathways & References \\
\hline WASF3 & NA & Inducing & OXA & Decreasing & ATG12 & Nie et al., 2020 \\
\hline PLK1 & Up & Inducing & DDP & Decreasing & CDC25C, cyclin B1, LC3-I, LC3-II & Chen et al., 2019 \\
\hline AQP3 & Up & Inducing & DDP & Decreasing & Atg5, Beclin-1, P62 & Dong et al., 2016 \\
\hline DJ-1 & Up & Inducing & $\mathrm{EPI}$ & Decreasing & LC3-I, LC3-II & Pan et al., 2018 \\
\hline TSPAN9 & Up & Inducing & $5-\mathrm{FU}$ & Decreasing & $\mathrm{PI} 3 \mathrm{~K} / \mathrm{AKT} / \mathrm{mTOR}$ & Qi et al., 2020 \\
\hline CD133 & Up & Inducing & DDP & Decreasing & $\mathrm{PI3K} / \mathrm{AKT} / \mathrm{mTOR}$ & Lu et al., 2019 \\
\hline TRIM14 & Up & Inducing & 5-FU, OXA & Decreasing & AMPK/mTOR & Xiao et al., 2020 \\
\hline $\mathrm{MTDH}$ & Up & Inducing & $5-\mathrm{FU}$ & Decreasing & AMPK/ATG5 & Pei et al., 2018 \\
\hline ERAS & Up & Inhibiting & DDP & Decreasing & AKT/mTOR & Tian et al., 2019 \\
\hline CCL2 & Up & Inhibiting & DDP & Decreasing & PI3K/AKT/mTOR, SQSTM1 & Xu et al., 2018b \\
\hline RAB5A & Up & Inhibiting & DDP & Decreasing & mTOR & Xu et al., 2018a \\
\hline MGMT & NA & Inhibiting & DDP & Increasing & ATG4B & Lei et al., 2020 \\
\hline CISD2 & Down & Inhibiting & $5-\mathrm{FU}$ & Increasing & AKT/mTOR & Sun et al., 2017 \\
\hline
\end{tabular}

5-FU, 5-fluorouracil; DDP, cisplatin; EPI, epirubicin; GC, gastric cancer; OXA, oxaliplatin; NA, not applicable.

TABLE 2 | Key regulatory non-coding RNAs in autophagy-mediated chemoresistance of gastric cancer.

\begin{tabular}{|c|c|c|c|c|c|c|}
\hline Biomarker & Expression in GC & Effects on autophagy & Chemotherapy & $\begin{array}{c}\text { Effects on } \\
\text { chemosensitivity }\end{array}$ & $\begin{array}{l}\text { Downstream } \\
\text { pathways }\end{array}$ & References \\
\hline miR-874 & Down & Inhibiting & DDP & Increasing & ATG16L1 & Huang et al., 2018 \\
\hline miR-30a & Down & Inhibiting & DDP & Increasing & MDR1, P-gp & Du et al., 2018 \\
\hline miR-181a & Down & Inhibiting & DDP & Increasing & MTMR3 & Lin et al., 2017 \\
\hline miR-23b-3p & Down & Inhibiting & DDP, VCR, 5-FU & Increasing & ATG12, HMGB2 & An et al., 2015 \\
\hline miR-148a-3p & Down & Inhibiting & DDP & Increasing & RAB12, mTORC1 & Li et al., 2017 \\
\hline miR-495-3p & Down & Inhibiting & ADM, DDP, 5-FU, VCR & Increasing & GRP78/mTOR & Chen et al., 2018 \\
\hline $\operatorname{miR}-361-5 p$ & NA & Inhibiting & DOC & Increasing & $\begin{array}{c}\mathrm{PI} 3 \mathrm{~K} / \mathrm{AKT} / \mathrm{mTOR}, \\
\text { FOXM1 }\end{array}$ & Tian et al., 2018 \\
\hline $\operatorname{miR}-21$ & NA & Inhibiting & DDP & Decreasing & $\begin{array}{l}\mathrm{PI} 3 \mathrm{~K} / \mathrm{AKT} / \mathrm{mTOR}, \\
\text { Beclin-1, LC3 }\end{array}$ & Gu et al., 2020 \\
\hline HOTTIP & Up & Inhibiting & DDP & Decreasing & $\begin{array}{c}\text { miR-216a-5p, BCL-2, } \\
\text { Beclin-1 }\end{array}$ & Zhao et al., 2020 \\
\hline MALAT1 & Up & Inducing & DDP, VCR & Decreasing & $\begin{array}{c}\text { miR-30b, ATG5, } \\
\text { miR-23b-3p, ATG12 }\end{array}$ & $\begin{array}{c}\text { YiRen et al., 2017; Xi } \\
\text { et al., } 2019\end{array}$ \\
\hline HULC & NA & Inducing & DDP & Decreasing & FoxM1 & Xin et al., 2019 \\
\hline
\end{tabular}

5-FU, 5-fluorouracil; ADM, adriamycin; DDP, cisplatin; DOC, docetaxel; GC, gastric cancer; VCR, vincristine; NA, not applicable. 

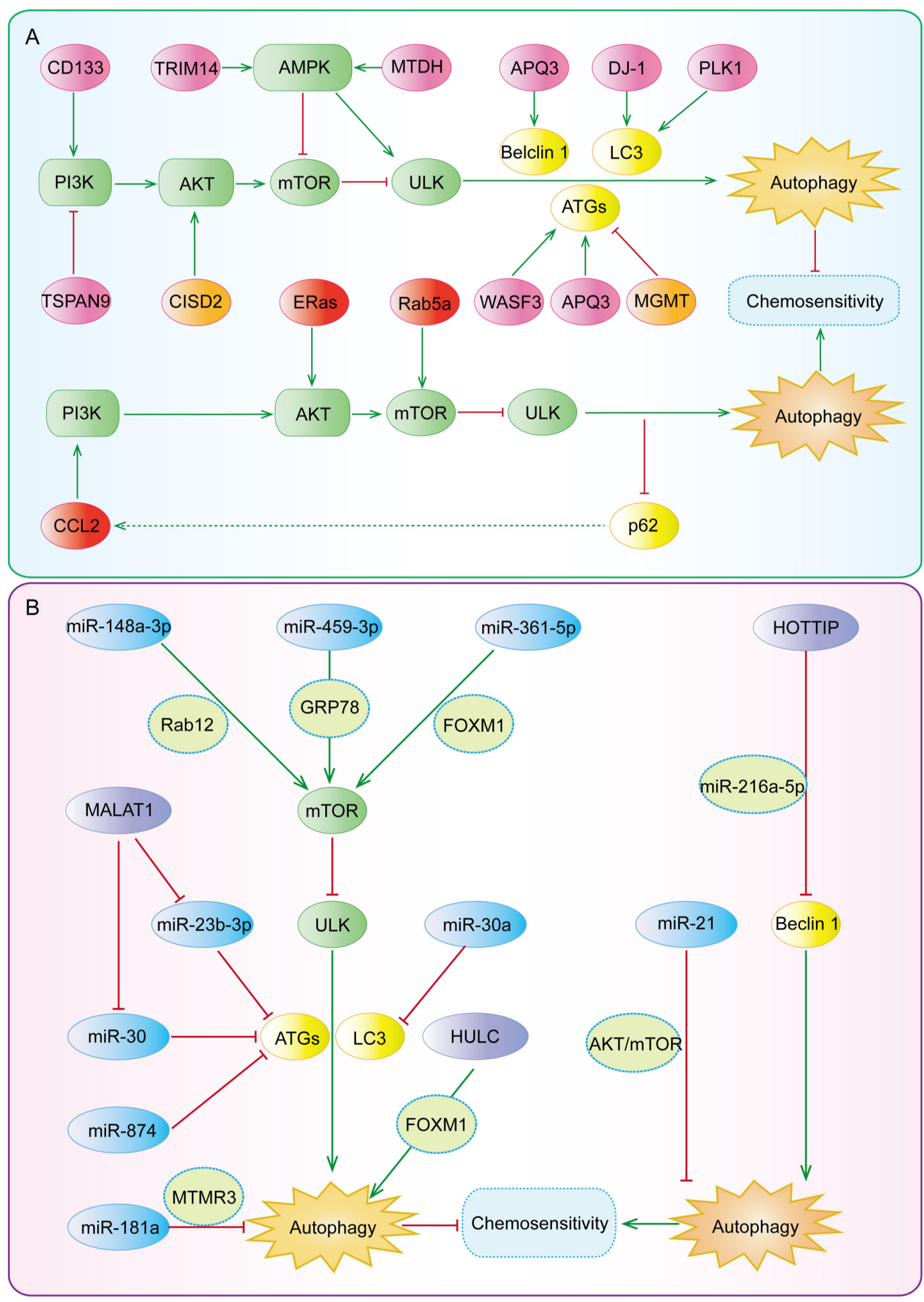

FIGURE 5 | The key regulatory nodes in autophagy-mediated gastric cancer chemoresistance. Various regulatory nodes, including several (A) proteins and (B) ncRNAs, are involved in the regulation of autophagy, thereby enhancing or reversing the chemotherapy resistance of gastric cancer.

CDGSH iron sulfur domain 2 (CISD2) is a survival gene and plays an important role in the redox reaction, longevity, tumorigenesis, and tumor progression (Li et al., 2019;
Nobili et al., 2020). Sun et al. (2017) have shown that CISD2 is significantly down-regulated in gastric cancer tissues and drug-resistant cell lines, which contributes to the resistance of 
gastric cancer cells to 5-FU. Further studies have found that CISD2 overexpression antagonizes 5-FU-induced autophagy by increasing the phosphorylation levels of AKT (S473) and mTOR (Ser2448), consequently reversing the reduction of p-MTOR and p-AKT by 5-FU and enhancing the sensitivity of MKN1 and BGC823 cells to 5-FU (Sun et al., 2017).

\section{Regulatory Effects of miRNAs on Autophagy-Mediated Gastric Cancer Chemoresistance}

MiRNAs, as a kind of highly conserved short ncRNAs, have a length of 18-25 nucleotides, regulate the expression of many genes, and participate in the development of human cancer and drug resistance (Poursheikhani et al., 2020; Ratti et al., 2020). miRNA-mediated autophagy (Figure 5B) has been found to play a role in chemoresistance in gastric cancer by regulating autophagy-related genes or signaling pathways (Jamali et al., 2020).

To date, many miRNAs, including miR-874, miR-30, miR181a, miR-23b-3p, miR-148a-3p, miR-495-3p, miR-361-5p, and miR-21 have been involved in the regulation of autophagy and chemoresistance in gastric cancer (as summarized in Table 2). Among them, the expression of miR-874, miR-30, miR-181a, and miR-23b-3p are down-regulated in drug-resistant gastric cancer cell lines and gastric cancer tissues (An et al., 2015; Lin et al., 2017; Du et al., 2018; Huang et al., 2018). Huang et al. (2018) have found that miR-874 inhibits autophagy by down-regulating ATG16L1, thus enhancing the sensitivity of gastric cancer cells to DDP in vitro and in vivo. Du et al. (2018) have reported that miR-30a up-regulates the expression of P-gp and MDR1, decreases the LC3-II/I ratio, inhibits autophagy, and reverses DDP resistance. Lin et al. (2017) have shown that miR-181a directly targets the $3^{\prime}$-UTR of myotubularin related protein 3 (MTMR3), decreases the mRNA and protein levels of MTMR3, inhibits autophagy, and increases the sensitivity of gastric cancer cells to DDP. An et al. (2015) have demonstrated that miR-23b-3p directly targets ATG12 and high-mobility group box 2 (HMGB2), inhibits autophagy, and increases the sensitivity of gastric cancer cells to DDP, vincristine (VCR), and 5-FU.

It has been found that both miR-148a-3p and miR-495$3 p$ are lowly expressed in drug-resistant gastric cancer cell lines and tumor tissues (Li et al., 2017; Chen et al., 2018). Li et al. (2017) have found that miR-148a-3p down-regulates the expression of A-kinase anchoring protein 1 (AKAP1) and RAB12 (RAB12, member RAS oncogene family) and reduces the inhibitory effects of RAB12 on mTORC1, thereby inhibiting autophagy and reversing the resistance of gastric cancer cells to DDP. Chen et al. (2018) have reported that miR-495-3p directly targets glucose regulated protein 78 (GRP78) at the post-transcriptional level, activates mTOR signaling, inhibits autophagy, and reverses multidrug resistance (MDR) in gastric cancer. Tian et al. (2018) have suggested that miR-361-5p negatively regulates the expression of FOXM1, which further causes an increase in the expression of p-AKT and mTOR, the inhibition of autophagy, and the enhanced sensitivity of gastric cancer cells to Docetaxel (DOC) in vitro. Gu et al. (2020) have found that miR-21 overexpression can increase the phosphorylation of AKT and mTOR, inhibit autophagy, and induce DDP resistance. It has been further demonstrated that the knockdown of miR-21 can promote autophagy and then sensitize DDP-resistant gastric cancer cell lines to DDP (Gu et al., 2020).

\section{Regulatory Effects of IncRNAs on Autophagy-Mediated Gastric Cancer Chemoresistance}

LncRNAs are the transcripts of more than 200 nucleotides, accounting for $80-90 \%$ of all ncRNAs, and regulate gene expression at the pre-transcriptional, transcriptional, and

TABLE 3 | Modulatory effects of autophagy inhibitor and activators on gastric cancer chemoresistance.

\begin{tabular}{|c|c|c|c|c|c|c|}
\hline Compounds & Source & Effects on autophagy & Chemotherapy & $\begin{array}{c}\text { Effects on } \\
\text { chemosensitivity }\end{array}$ & $\begin{array}{l}\text { Downstream } \\
\text { pathways }\end{array}$ & References \\
\hline Genipin & $\begin{array}{c}\text { Natural products from } \\
\text { TM }\end{array}$ & Inducing & OXA & Increasing & p53, DRAM & Kim et al., 2020 \\
\hline Tanshinone IIA & $\begin{array}{c}\text { Natural products from } \\
\text { TM }\end{array}$ & Inducing & ADM & Increasing & LC3-II, P62 & Xu Z. et al., 2018 \\
\hline Liquiritin & $\begin{array}{c}\text { Natural products from } \\
\text { TM }\end{array}$ & Inducing & DDP & Increasing & LC3B, Beclin-1, p62 & Wei et al., 2017 \\
\hline DSGOST & Prescription from TM & Inducing & DDP & Increasing & $\begin{array}{l}\text { LC3-II, ATG5, Beclin-1, } \\
\text { Bcl2, AMPK } \alpha / \text { ULK1 }\end{array}$ & Kim T. W. et al., 2018 \\
\hline Cucurbitacin B & $\begin{array}{l}\text { Natural products from } \\
\text { edible plants }\end{array}$ & Inducing & DDP & Increasing & CIP2A/PP2A/mTORC1 & Dandawate et al., 2020 \\
\hline Phloretin & $\begin{array}{l}\text { Natural products from } \\
\text { edible plants }\end{array}$ & Inducing & ADM & Increasing & $\begin{array}{c}\text { ERK1/2, MAP, LC3B II, } \\
\text { Beclin-1 }\end{array}$ & You et al., 2020 \\
\hline Tunicamycin & Antibiotic & Inducing & ADM, VCR & Increasing & LC3-I, LC3-II & Wu et al., 2018 \\
\hline Indomethacin & NSAIDs & Inhibiting & OXA & Increasing & $\begin{array}{c}\text { LC3-II, LC3-II, p62, } \\
\text { NBR1 }\end{array}$ & $\begin{array}{c}\text { Vallecillo-Hernandez } \\
\text { et al., } 2018\end{array}$ \\
\hline Propofol & Anesthetic & Inhibiting & DDP & Increasing & $\begin{array}{c}\text { MALAT1, miR-30e, } \\
\text { ATG5 }\end{array}$ & Zhang Y.F. et al., 2020 \\
\hline
\end{tabular}

ADM, adriamycin; DDP, cisplatin; OXA, oxaliplatin; TM, traditional medicine; VCR, vincristine. 
post-transcriptional levels (Yuan et al., 2020). Therefore, lncRNAs widely participate in various physiological and pathological processes of organisms (Yuan et al., 2020). LncRNAs have been found to regulate autophagy and drug resistance (Figure 5B) by acting as a competing endogenous RNA (ceRNA) or directly binding to proteins to modulate their expression and functions (as summarized in Table 2).

It has been found that the expression levels of HOTTIP (HOXA distal transcript antisense RNA), MALAT1 (metastasis associated lung adenocarcinoma transcript 1), and HULC (hepatocellular carcinoma up-regulated long non-coding RNA) are elevated in chemotherapy-resistant gastric cancer cell lines and tumor tissues (YiRen et al., 2017; Xin et al., 2019; Zhao et al., 2020). Zhao et al. (2020) have indicated that HOTTIP may function as a ceRNA of miR-216a-5p, increasing the expression level of BCL-2 and decreasing the expression level of Beclin1. Further studies have shown that HOTTIP overexpression inhibits autophagy and induces DDP resistance in gastric cancer while the silencing of HOTTIP increases the sensitivity of gastric cancer cells to DDP (Zhao et al., 2020). YiRen et al. (2017) have reported that MALAT1 acts as a ceRNA against miR$23 b-3 p$ and attenuates the inhibitory effects of miR-23b-3p on ATG12, leading to autophagy induction and VCR resistance in gastric cancer cells. Moreover, Xi et al. (2019) have found that MALAT1 can competitively bind to miR-30 and promote the expression of ATG5, thus inducing autophagy and DDP resistance. Xin et al. (2019) have shown that HULC can stabilize FoxM1 by inhibiting its ubiquitination and induce autophagy and DDP resistance. It has further been observed that intervening HULC can suppress autophagy and then reduce the resistance of gastric cancer cells to DDP in vitro (Xin et al., 2019).

\section{MODULATORY EFFECTS OF AUTOPHAGY INHIBITORS AND ACTIVATORS ON GASTRIC CANCER CHEMORESISTANCE}

Given the importance of autophagy in chemotherapy resistance, the development of autophagy inhibitors or activators may provide a new opportunity for the treatment of human cancer, especially for those with drug resistance (Mele et al., 2020). FDA has approved the clinical application of CQ and its derivative hydroxychloroquine (HCQ), which can inhibit autophagy by blocking autophagosome fusion and degradation (Liu T. et al., 2020a). At present, there are several clinical studies on the treatment of multiple tumors with HCQ alone or in combination with chemotherapy. Various natural products from TM, medicinal plants, or microorganisms, such as flavonoids, alkaloids, terpenoids, coumarins, etc. have been reported as potential autophagy inhibitors and activators and MDR reversal agents (as summarized in Table 3) (Sato et al., 2019; Liu S. et al., 2020; Nazim et al., 2020).

\section{Effects of Autophagy Activators on Gastric Cancer Chemoresistance}

Genipin, a natural product derived from traditional Chinese medicine (TCM) Gardenia jasminoides, has shown antiangiogenic, anti-proliferative, anti-inflammatory, and anticancer activities (Jo et al., 2019; Zhang C. et al., 2020; Zhou et al., 2020). Kim et al. (2020) have found that genipin can increase the expression of p53 and DRAM, induce apoptosis and autophagy, and enhance the sensitivity of AGS and MKN45 gastric cancer cell lines to OXA. Tanshinones are a group of abietane diterpenes from the TCM Danshen and have exhibited anticancer activities in vitro and in vivo (Li M. et al., 2020). Xu Z. et al. (2018) have reported that tanshinone IIA can reduce the expression of multidrug resistance protein 1 (MRP1) and inhibit the efflux of adriamycin (ADM). Besides, the combination of tanshinone IIA with $\mathrm{ADM}$ can induce autophagy, thus promoting cell apoptosis and enhancing the sensitivity of gastric cancer cells to ADM (Xu Z. et al., 2018). Liquiritin is the main medicinal component of TCM licorice. Wei et al. (2017) have found that liquiritin increases Beclin-1 expression, inhibits p62 expression, triggers autophagy and apoptosis, and then reverses DDP resistance in vitro and in vivo.

Danggui-Sayuk-Ga-Osuyu-Saenggang-Tang (DSGOST) is a traditional Korean herbal medicine (Kim T. W. et al., 2018). DSGOST is similar to TCM Danggui-Sini-Jia-WuzhuyuShengjian-Tang and is commonly used in treating patients with Raynaud's phenomenon caused by multiple chemotherapy drugs (Kim T. W. et al., 2018). Kim T. W. et al. (2018) have reported that DSGOST can cause the dissociation of the Beclin$1-\mathrm{Bcl} 2$ complex, activate the AMPK/ULK1 pathway, increase the autophagy flux, induce autophagy and apoptosis, and enhance the sensitivity of gastric cancer cell lines AGS and SNU-638 to DDP.

The dietary natural products, cucurbitacin B and phloretin have recently been found to exert anticancer activities and reverse chemotherapy resistance (Hsiao et al., 2019; Dandawate et al., 2020). Xu et al. (2020) have shown that cucurbitacin B induces autophagy and apoptosis and reverses the sensitivity of DDP-resistant cells to DDP. The mechanisms of action studies have indicated that cucurbitacin $\mathrm{B}$ inhibits CIP2A (cancerous inhibitor of protein phosphatase $2 \mathrm{~A}$ ), subsequently reactivating PP2A (protein phosphatase $2 \mathrm{~A}$ ) and enhancing PP2A-dependent mTORC1 inactivation (Xu et al., 2020). You et al. (2020) have found that phloretin inhibits the phosphorylation of ERK1/2 and MAPK p38 and increases the expression of LC3B II and Beclin-1, thus inducing autophagy and enhancing the sensitivity of gastric cancer cells to ADM in vitro.

Abnormal glycosylation has been widely regarded as an important sign of cancer and is significantly associated with tumor development, progression, metastasis, and chemoresistance (Liu et al., 2018; Liu T. et al., 2020a). As an effective glycosylation inhibitor, tunicamycin has been initially identified as a natural antibiotic and anticancer compound (Liu Y. C. et al., 2020b; Santos-Laso et al., 2020). Wu et al. (2018) have suggested that tunicamycin inhibits $N$-glycosylation to aggravate ER stress, induces autophagy, and increases the sensitivity of gastric cancer cells to ADM and VCR. 


\section{Effects of Autophagy Inhibitors on Gastric Cancer Chemoresistance}

Indomethacin, a common non-steroidal anti-inflammatory drug (NSAID), has been reported as a coadjutant of anticancer drugs with satisfactory efficacy (López-Contreras et al., 2019; Seetha et al., 2020). Vallecillo-Hernandez et al. (2018) have found that indomethacin can induce the accumulation of p62 and neighbor of BRCA1 (NBR1), impair lysosomal function, inhibit autophagic degradation, and increase OXA-induced cell death in AGS cells. Propofol, a sedative widely used in surgery, has shown efficacy in several types of cancer, such as pancreatic cancer (Wang et al., 2020), gastric cancer (Liu F. et al., 2020), colon cancer (Liu F. et al., 2020), papillary thyroid carcinoma (Li Y. et al., 2020), and so on. Zhang et al. have demonstrated that propofol in combination with DDP inhibits the expression of lncRNA MALAT1 and enhances the inhibitory effects of miR-30e on ATG5 and autophagy, thereby increasing the sensitivity of gastric cancer cells to DDP in vivo and in vitro (Zhang Y.F. et al., 2020).

\section{CONCLUSION AND FUTURE DIRECTION}

Overall, this review provides compelling evidence for the dual roles of autophagy in the chemoresistance of gastric cancer. Autophagy can protect cancer cells from chemotherapy and participate in the formation of MDR, while it can also promote apoptosis and kill MDR cancer cells. Therefore, the development of autophagy inhibitors or activators may be an important way to reverse drug resistance and enhance chemosensitivity. The combination of autophagy modulators and chemotherapy drugs will also bring new hope for the treatment of human cancer. For instance, it is exciting to note that the combination of autophagic inhibitor HCQ and gemcitabine or nab-paclitaxel can significantly improve the overall response rate of cancer patients (Karasic et al., 2019).

The dual roles of autophagy in drug resistance are mainly regulated by different proteins and ncRNAs. Proteins and ncRNAs can monitor autophagy by regulating autophagy regulatory proteins and/or autophagy regulatory signaling pathways. In the fields of drug resistance and autophagy in gastric cancer, researchers have focused on PI3K/AKT and MAPK pathways. More studies on other autophagy-related pathways (p53, MAPK, or PTEN pathways) are expected in the future. The development of small-molecule inhibitors or activators targeting key regulatory nodes in the process of autophagy may provide an alternative treatment for patients with cancer. For example, SBI-0206965, as a small molecule inhibitor of ULK1, has been

\section{REFERENCES}

Abdrakhmanov, A., Gogvadze, V., and Zhivotovsky, B. (2020). To eat or to die: deciphering selective forms of autophagy. Trends Biochem. Sci. 45, 347-364. doi: 10.1016/j.tibs.2019.11.006

Abdu, K., Aiertza, M. K., Wilkinson, O. J., Senthong, P., Craggs, T. D., Povey, A. C., et al. (2020). Synthesis of oligodeoxyribonucleotides containing a tricyclic thio analogue of $\mathrm{O}(6)$-methylguanine and their recognition by MGMT and found to enhance daunorubicin sensitivity in acute myeloid leukemia (Qiu et al., 2020). Besides, the ULK1 activator LYN1604, which can induce autophagy-related cell death through the ULK complex, shows significant anticancer activity in triple-negative breast cancer (Ouyang et al., 2017). Therefore, further strengthening the research on the role of different proteins and ncRNAs in regulating autophagy-mediated gastric cancer chemoresistance will be beneficial to the development of promising drugs for gastric cancer therapy.

Many natural products from TM and edible plants have shown preventive and therapeutic efficacy in human cancer by targeting multiple signaling pathways and inducing cell cycle arrest and apoptosis (Koh et al., 2020). Accumulating evidence has demonstrated the modulatory effects of these anticancer natural products on autophagy and chemosensitivity. Thus, using natural products alone or in combination with autophagy modulators and/or chemotherapy drugs may exhibit promising efficacy in human cancer, especially in drug-resistant cancer. Nevertheless, further studies are warranted to identify the specific molecular targets of these natural products and examine the efficacy and safety of these strategies in clinically relevant cancer models.

\section{AUTHOR CONTRIBUTIONS}

J-JQ and X-DC conceptualized the manuscript. J-LX, LY, Y-CT, $\mathrm{Z}-\mathrm{YX}$, and $\mathrm{H}-\mathrm{DX}$ collected the literature, wrote the manuscript, and made the figures. J-JQ edited and made significant revisions to the manuscript. All authors read and approved the final manuscript.

\section{FUNDING}

This study was supported by Natural Science Foundation of Zhejiang Province (LY18H290006), National Natural Science Foundation of China (81903842 and 81973634), and Program of Zhejiang Provincial TCM Sci-tech Plan (2018ZY006 and 2020ZZ005).

\section{ACKNOWLEDGMENTS}

We thank the current and former members of our laboratories and collaborators for their contributions to the publications cited in this review article. The research field in autophagy is rapidly growing, and we apologize for not being able to cite all the recent publications, due to space limitation.

Atl1. Nucleos. Nucleot. Nucl. Acids 39, 1108-1121. doi: 10.1080/15257770.2020. 1764971

Amaravadi, R., Kimmelman, A., and Debnath, J. (2019). Targeting autophagy in cancer: recent advances and future directions. Cancer Discov. 9, 1167-1181. doi: 10.1158/2159-8290.Cd-19-0292

An, Y., Zhang, Z., Shang, Y., Jiang, X., Dong, J., Yu, P., et al. (2015). miR-23b-3p regulates the chemoresistance of gastric cancer cells by targeting ATG12 and HMGB2. Cell Death Dis. 6:e1766. doi: 10.1038/cddis.2015.123 
Anerillas, C., Abdelmohsen, K., and Gorospe, M. (2020). Regulation of senescence traits by MAPKs. Geroscience 42, 397-408. doi: 10.1007/s11357-020-00183-3

Bakula, D., Müller, A., Zuleger, T., Takacs, Z., Franz-Wachtel, M., Thost, A., et al. (2017). WIPI3 and WIPI $4 \beta$-propellers are scaffolds for LKB1-AMPKTSC signalling circuits in the control of autophagy. Nat. Commun. 8:15637. doi: 10.1038/ncomms 15637

Balvers, R. K., Lamfers, M. L., Kloezeman, J. J., Kleijn, A., Berghauser Pont, L. M., Dirven, C. M., et al. (2015). ABT-888 enhances cytotoxic effects of temozolomide independent of MGMT status in serum free cultured glioma cells. J. Transl. Med. 13:74. doi: 10.1186/s12967-015-0427-y

Barroso-Chinea, P., Luis-Ravelo, D., Fumagallo-Reading, F., Castro-Hernandez, J., Salas-Hernandez, J., Rodriguez-Nuñez, J., et al. (2020). DRD3 (dopamine receptor D3) but not DRD2 activates autophagy through MTORC1 inhibition preserving protein synthesis. Autophagy 16, 1279-1295. doi: 10.1080/15548627. 2019.1668606

Biagioni, A., Skalamera, I., Peri, S., Schiavone, N., Cianchi, F., Giommoni, E., et al. (2019). Update on gastric cancer treatments and gene therapies. Cancer Metast. Rev. 38, 537-548. doi: 10.1007/s10555-019-09803-7

Boosani, C., Gunasekar, P., and Agrawal, D. K. (2019). An update on PTEN modulators - a patent review. Exp. Opin. Ther. Pat. 29, 881-889. doi: 10.1080/ 13543776.2019 .1669562

Cai, C. Z., Yang, C., Zhuang, X. X., Yuan, N., Wu, M.-Y., Tan, J.-Q., et al. (2020). NRBF2 is a RAB7 effector required for autophagosome maturation and mediates the association of APP-CTFs with active form of RAB7 for degradation. Autophagy 1-19. doi: 10.1080/15548627.2020.1760623

Cai, J., Li, R., Xu, X., Zhang, L., Lian, R., Fang, L., et al. (2018). CK1 $\alpha$ suppresses lung tumour growth by stabilizing PTEN and inducing autophagy. Nat. Cell Biol. 20, 465-478. doi: 10.1038/s41556-018-0065-8

Cao, Y., Luo, Y., Zou, J., Ouyang, J., Cai, Z., Zeng, X., et al. (2019). Autophagy and its role in gastric cancer. Clin. Chim. Acta 489, 10-20. doi: 10.1016/j.cca.2018. 11.028

Carling, D. (2017). AMPK signalling in health and disease. Curr. Opin. Cell Biol. 45, 31-37. doi: 10.1016/j.ceb.2017.01.005

Chen, J., Zhang, P., Chen, W., Li, D., Wu, X., Deng, R., et al. (2015). ATM-mediated PTEN phosphorylation promotes PTEN nuclear translocation and autophagy in response to DNA-damaging agents in cancer cells. Autophagy 11, 239-252. doi: $10.1080 / 15548627.2015 .1009767$

Chen, S., Wu, J., Jiao, K., Wu, Q., Ma, J., Chen, D., et al. (2018). MicroRNA-495-3p inhibits multidrug resistance by modulating autophagy through GRP78/mTOR axis in gastric cancer. Cell Death Dis. 9:1070. doi: 10.1038/s41419-018-0950-x

Chen, Z., Chai, Y., Zhao, T., Li, P., Zhao, L., He, F., et al. (2019). Effect of PLK1 inhibition on cisplatin-resistant gastric cancer cells. J. Cell Physiol. 234, 5904-5914. doi: 10.1002/jcp.26777

Cheng, C. Y., Tseng, H. H., Chiu, H. C., Chang, C. D., Nielsen, B. L., and Liu, H. J. (2019). Bovine ephemeral fever virus triggers autophagy enhancing virus replication via upregulation of the Src/JNK/AP1 and PI3K/Akt/NF-кB pathways and suppression of the PI3K/Akt/mTOR pathway. Vet. Res. 50:79. doi: 10.1186/s13567-019-0697-0

Chi, C., Leonard, A., Knight, W., Beussman, K., Zhao, Y., Cao, Y., et al. (2019). LAMP-2B regulates human cardiomyocyte function by mediating autophagosome-lysosome fusion. Proc. Natl. Acad. Sci. U.S.A. 116, 556-565. doi: $10.1073 /$ pnas. 1808618116

Chollat-Namy, M., Ben Safta-Saadoun, T., Haferssas, D., Meurice, G., Chouaib, S., Thiery, J., et al. (2019). The pharmalogical reactivation of p53 function improves breast tumor cell lysis by granzyme B and NK cells through induction of autophagy. Cell Death Dis. 10:695. doi: 10.1038/s41419-019-1950-1

Corona, A. K., and Jackson, W. T. (2018). Finding the middle ground for autophagic fusion requirements. Trends Cell Biol. 28, 869-881. doi: 10.1016/j. tcb.2018.07.001

Crighton, D., Wilkinson, S., O'Prey, J., Syed, N., Smith, P., Harrison, P., et al. (2006). DRAM, a p53-induced modulator of autophagy, is critical for apoptosis. Cell 126, 121-134. doi: 10.1016/j.cell.2006. 05.034

Dandawate, P., Subramaniam, D., Panovich, P., Standing, D., Krishnamachary, B., Kaushik, G., et al. (2020). Cucurbitacin B and I inhibits colon cancer growth by targeting the Notch signaling pathway. Sci. Rep. 10:1290. doi: 10.1038/s41598020-57940-9
Diao, J., Liu, R., Rong, Y., Zhao, M., Zhang, J., Lai, Y., et al. (2015). ATG14 promotes membrane tethering and fusion of autophagosomes to endolysosomes. Nature 520, 563-566. doi: 10.1038/nature 14147

Dong, X., Wang, Y., Zhou, Y., Wen, J., Wang, S., and Shen, L. (2016). Aquaporin 3 facilitates chemoresistance in gastric cancer cells to cisplatin via autophagy. Cell Death Discov. 2:16087. doi: 10.1038/cddiscovery.2016.87

Du, X., Liu, B., Luan, X., Cui, Q., and Li, L. (2018). miR-30 decreases multidrug resistance in human gastric cancer cells by modulating cell autophagy. Exp. Ther. Med. 15, 599-605. doi: 10.3892/etm.2017.5354

Dutta, P., Paico, K., Gomez, G., Wu, Y., and Vadgama, J. V. (2020). Transcriptional Regulation of CCL2 by PARP1 is a driver for invasiveness in breast cancer. Cancers 12, 1317. doi: 10.3390/cancers12051317

Dutta, S., Mahalanobish, S., Saha, S., Ghosh, S., and Sil, P. C. (2019). Natural products: an upcoming therapeutic approach to cancer. Food Chem. Toxicol. 128, 240-255. doi: 10.1016/j.fct.2019.04.012

Emanuele, S., Notaro, A., Palumbo Piccionello, A., Maggio, A., Lauricella, M. D’Anneo, A., et al. (2018). Sicilian litchi fruit extracts induce autophagy versus apoptosis switch in human colon cancer cells. Nutrients 10:1490. doi: 10.3390/ nu10101490

Fan, J., Liu, Y., Yin, J., Li, Q., Li, Y., Gu, J., et al. (2016). Oxygen-GlucoseDeprivation/Reoxygenation-induced autophagic cell death depends on JNKmediated phosphorylation of Bcl-2. Cell Physiol. Biochem. 38, 1063-1074. doi: $10.1159 / 000443057$

Fawal, M. A., Brandt, M., and Djouder, N. (2015). MCRS1 binds and couples Rheb to amino acid-dependent mTORC1 activation. Dev. Cell 33, 67-81. doi: 10.1016/j.devcel.2015.02.010

Fitzmaurice, C., Abate, D., Abbasi, N., Abbastabar, H., Abd-Allah, F., AbdelRahman, O., et al. (2019). Global, regional, and national cancer incidence, mortality, years of life lost, years lived with disability, and disability-adjusted life-years for 29 cancer groups, 1990 to 2017: a systematic analysis for the global burden of disease study. JAMA Oncol. 5, 1749-1768. doi: 10.1001/jamaoncol. 2019.2996

Galluzzi, L., and Green, D. R. (2019). Autophagy-independent functions of the autophagy machinery. Cell 177, 1682-1699. doi: 10.1016/j.cell.2019.05.026

Gao, A., Li, F., Zhou, Q., and Chen, L. (2020). Sestrin2 as a potential therapeutic target for cardiovascular diseases. Pharmacol. Res. 159:104990. doi: 10.1016/j. phrs.2020.104990

Gao, S., Zhao, Z., Wu, R., Wu, L., Tian, X., and Zhang, Z. (2018). MiR-146b inhibits autophagy in prostate cancer by targeting the PTEN/Akt/mTOR signaling pathway. Aging 10, 2113-2121. doi: 10.18632/aging.101534

Gomez-Osuna, A., Calatrava, V., Galvan, A., Fernandez, E., and Llamas, A. (2020). Identification of the MAPK Cascade and its Relationship with Nitrogen Metabolism in the Green Alga Chlamydomonas reinhardtii. Int. J. Mol. Sci. 21:3417. doi: 10.3390/ijms21103417

Gu, Y., Fei, Z., and Zhu, R. (2020). miR-21 modulates cisplatin resistance of gastric cancer cells by inhibiting autophagy via the PI3K/Akt/mTOR pathway. Anticancer Drugs 31, 385-393. doi: 10.1097/cad.0000000000000886

Guardia, C., Tan, X., Lian, T., Rana, M., Zhou, W., Christenson, E., et al. (2020). Structure of human ATG9A, the only transmembrane protein of the core autophagy machinery. Cell Rep. 31:107837. doi: 10.1016/j.celrep.2020.107837

Hafner, A., Bulyk, M., Jambhekar, A., and Lahav, G. (2019). The multiple mechanisms that regulate p53 activity and cell fate. Nat. Rev. Mol. Cell Biol. 20, 199-210. doi: 10.1038/s41580-019-0110-x

Hao, Q., Vadgama, J. V., and Wang, P. (2020). CCL2/CCR2 signaling in cancer pathogenesis. Cell Commun. Signal. 18:82. doi: 10.1186/s12964-020-00589-8

Hempen, C. H., and Hummelsberger, J. (2020). [Traditional Chinese medicine (TCM)-what is myth and what is the state of evidence today?]. Bundesgesundheitsblatt Gesundheitsforschung Gesundheitsschutz 63, 570-576. doi: 10.1007/s00103-020-03132-9

Hollenstein, D. M., and Kraft, C. (2020). Autophagosomes are formed at a distinct cellular structure. Curr. Opin. Cell Biol. 65, 50-57. doi: 10.1016/j.ceb.2020.02. 012

Høyer-Hansen, M., and Jäättelä, M. (2008). Autophagy: an emerging target for cancer therapy. Autophagy 4, 574-580. doi: 10.4161/auto.5921

Hsiao, Y. H., Hsieh, M. J., Yang, S. F., Chen, S. P., Tsai, W. C., and Chen, P. N. (2019). Phloretin suppresses metastasis by targeting protease and inhibits cancer stemness and angiogenesis in human cervical 
cancer cells. Phytomedicine 62:152964. doi: 10.1016/j.phymed.2019.15 2964

Hu, W., Chen, S., Thorne, R., and Wu, M. (2019). TP53. TP53 Target Genes (DRAM, TIGAR), and Autophagy. Adv. Exp. Med. Biol. 1206, 127-149. doi: 10.1007/978-981-15-0602-4_6

Huang, H., Tang, J., Zhang, L., Bu, Y., and Zhang, X. (2018). miR-874 regulates multiple-drug resistance in gastric cancer by targeting ATG16L1. Int. J. Oncol. 53, 2769-2779. doi: 10.3892/ijo.2018.4593

Jamali, Z., Taheri-Anganeh, M., Shabaninejad, Z., Keshavarzi, A., Taghizadeh, H., Razavi, Z. S., et al. (2020). Autophagy regulation by microRNAs: novel insights into osteosarcoma therapy. IUBMB Life 72, 1306-1321. doi: 10.1002/iub.2277

Jiang, H., Wang, H., Zou, W., Hu, Y., Chen, C., and Wang, C. (2019). Sufentanil impairs autophagic degradation and inhibits cell migration in NCI-H460 in vitro. Oncol. Lett. 18, 6829-6835. doi: 10.3892/ol.2019.10997

Jiang, S., Liu, Y., Xu, B., Zhang, Y., and Yang, M. (2020). Noncoding RNAs: New regulatory code in chondrocyte apoptosis and autophagy. Wiley Interdiscip. Rev. RNA 11, e1584. doi: 10.1002/wrna.1584

Jin, W. (2020). Novel Insights into PARK7 (DJ-1), a potential anti-cancer therapeutic target, and implications for cancer progression. J. Clin. Med. 9:1256. doi: $10.3390 /$ jcm 9051256

Jo, M. J., Jeong, S., Yun, H. K., Kim, D. Y., Kim, B. R., Kim, J. L., et al. (2019). Genipin induces mitochondrial dysfunction and apoptosis via downregulation of Stat3/mcl-1 pathway in gastric cancer. BMC Cancer 19:739. doi: 10.1186/ s12885-019-5957-x

Karasic, T. B., O'Hara, M. H., Loaiza-Bonilla, A., Reiss, K. A., Teitelbaum, U. R., Borazanci, E., et al. (2019). Effect of gemcitabine and nab-paclitaxel with or without hydroxychloroquine on patients with advanced pancreatic cancer: a phase 2 randomized clinical trial. JAMA Oncol. 5, 993-998. doi: 10.1001/ jamaoncol.2019.0684

Kim, B. R., Jeong, Y. A., Kim, D. Y., Kim, J. L., Jeong, S., Na, Y. J., et al. (2020). Genipin increases oxaliplatin-induced cell death through autophagy in gastric cancer. J. Cancer 11, 460-467. doi: 10.7150/jca.34773

Kim, B. W., Jin, Y., Kim, J., Kim, J. H., Jung, J., Kang, S., et al. (2018). The C-terminal region of ATG101 bridges ULK1 and PtdIns3K complex in autophagy initiation. Autophagy 14, 2104-2116. doi: 10.1080/15548627.2018. 1504716

Kim, T. W., Lee, S. Y., Kim, M., Cheon, C., Jang, B. H., Shin, Y. C., et al. (2018). DSGOST regulates resistance via activation of autophagy in gastric cancer. Cell Death Dis. 9:649. doi: 10.1038/s41419-018-0658-y

Kimmelman, A. C., and White, E. (2017). Autophagy and tumor metabolism. Cell Metab. 25, 1037-1043. doi: 10.1016/j.cmet.2017.04.004

Koh, Y. C., Ho, C. T., and Pan, M. H. (2020). Recent advances in cancer chemoprevention with phytochemicals. J. Food Drug Anal. 28, 14-37. doi: 10.1016/j.jfda.2019.11.001

Lei, Y., Tang, L., Hu, J., Wang, S., Liu, Y., Yang, M., et al. (2020). Inhibition of MGMT-mediated autophagy suppression decreases cisplatin chemosensitivity in gastric cancer. Biomed Pharmacother 125:109896. doi: 10.1016/j.biopha.2020. 109896

Levine, B., and Kroemer, G. (2019). Biological functions of autophagy genes: a disease perspective. Cell 176, 11-42. doi: 10.1016/j.cell.2018.09.048

Levy, J. M. M., Towers, C. G., and Thorburn, A. (2017). Targeting autophagy in cancer. Nat. Rev. Cancer 17, 528-542. doi: 10.1038/nrc.2017.53

Li, B., Wang, W., Li, Z., Chen, Z., Zhi, X., Xu, J., et al. (2017). MicroRNA-148a$3 p$ enhances cisplatin cytotoxicity in gastric cancer through mitochondrial fission induction and cyto-protective autophagy suppression. Cancer Lett. 410, 212-227. doi: 10.1016/j.canlet.2017.09.035

Li, J., Duan, H., Xuan, F., Zhao, E., and Huang, M. (2019). CDGSH iron sulfur domain 2 deficiency inhibits cell proliferation and induces cell differentiation of neuroblastoma. Pathol. Oncol. Res. 26, 1725-1733. doi: 10.1007/s12253-01900753-7

Li, M., Gao, F., Zhao, Q., Zuo, H., Liu, W., and Li, W. (2020). Tanshinone IIA inhibits oral squamous cell carcinoma via reducing Akt-c-Myc signalingmediated aerobic glycolysis. Cell Death Dis. 11:381. doi: 10.1038/s41419-0202579-9

Li, X., Yang, X., Liu, Y., Gong, N., Yao, W., Chen, P., et al. (2013). Japonicone A suppresses growth of Burkitt lymphoma cells through its effect on NFkappaB. Clin. Cancer Res. 19, 2917-2928. doi: 10.1158/1078-0432.CCR-123258
Li, Y., Wang, Y., Zou, M., Chen, C., Chen, Y., Xue, R., et al. (2018). AMPK blunts chronic heart failure by inhibiting autophagy. Biosci. Rep. 38:BSR20170982. doi: $10.1042 /$ bsr20170982

Li, Y., Zeng, Q. G., Qiu, J. L., Pang, T., Wang, H., and Zhang, X. X. (2020). Propofol suppresses migration, invasion, and epithelial-mesenchymal transition in papillary thyroid carcinoma cells by regulating miR-122 expression. Eur. Rev. Med. Pharmacol. Sci. 24, 5101-5110. doi: 10.26355/eurrev_202005_21203

Lin, Y., Zhao, J., Wang, H., Cao, J., and Nie, Y. (2017). miR-181a modulates proliferation, migration and autophagy in AGS gastric cancer cells and downregulates MTMR3. Mol. Med. Rep. 15, 2451-2456. doi: 10.3892/mmr. 2017.6289

Liu, B., Pan, S., Xiao, Y., Liu, Q., Xu, J., and Jia, L. (2018). LINC01296/miR26a/GALNT3 axis contributes to colorectal cancer progression by regulating O-glycosylated MUC1 via PI3K/AKT pathway. J. Exp. Clin. Cancer Res. 37:316. doi: 10.1186/s13046-018-0994- $\mathrm{x}$

Liu, F., Qiu, F., Fu, M., Chen, H., and Wang, H. (2020). Propofol reduces epithelial to mesenchymal transition. invasion and migration of gastric cancer cells through the MicroRNA-195-5p/Snail Axis. Med. Sci. Monit. 26:e920981. doi: $10.12659 / \mathrm{msm} .920981$

Liu, H., Ma, L., Lin, J., Cao, B., Qu, D., Luo, C., et al. (2020a). Advances in molecular mechanisms of drugs affecting abnormal glycosylation and metastasis of breast cancer. Pharmacol. Res. 155:104738. doi: 10.1016/j.phrs.2020.104738

Liu, H., Xie, S., Fang, F., Kalvakolanu, D. V., and Xiao, W. (2020b). SHQ1 is an ER stress response gene that facilitates chemotherapeutics-induced apoptosis via sensitizing ER-stress response. Cell Death Dis. 11:445. doi: 10.1038/s41419020-2656-0

Liu, S., Zhang, J., Yang, H., Zhang, Q., and Chen, M. (2020). Pectolinarigenin flavonoid exhibits selective anti-proliferative activity in cisplatin-resistant hepatocellular carcinoma, autophagy activation, inhibiting cell migration and invasion. G2/M phase cell cycle arrest and targeting ERK1/2 MAP kinases. J. Buon. 25, 415-420.

Liu, T., Zhang, J., Li, K., Deng, L., and Wang, H. (2020a). Combination of an autophagy inducer and an autophagy inhibitor: a smarter strategy emerging in cancer therapy. Front. Pharmacol. 11:408. doi: 10.3389/fphar.2020.00408

Liu, Y. C., Yeh, C. T., and Lin, K. H. (2020b). Cancer stem cell functions in hepatocellular carcinoma and comprehensive therapeutic strategies. Cells 9:1331. doi: 10.3390/cells9061331

López-Contreras, F., Muñoz-Uribe, M., Pérez-Laines, J., Ascencio-Leal, L., RiveraDictter, A., Martin-Martin, A., et al. (2019). Searching for drug synergy against cancer through polyamine metabolism impairment: insight into the metabolic effect of indomethacin on lung cancer cells. Front. Pharmacol. 10:1670. doi: 10.3389/fphar.2019.01670

Lőrincz, P., and Juhász, G. (2020). Autophagosome-lysosome fusion. J. Mol. Biol. 432, 2462-2482. doi: 10.1016/j.jmb.2019.10.028

Lu, R., Zhao, G., Yang, Y., Jiang, Z., Cai, J., and Hu, H. (2019). Inhibition of CD133 overcomes cisplatin resistance through inhibiting PI3K/AKT/mTOR signaling pathway and autophagy in CD133-positive gastric cancer cells. Technol. Cancer Res. Treat. 18:1533033819864311. doi: 10.1177/1533033819864311

Lu, Y., Dong, S., Hao, B., Li, C., Zhu, K., Guo, W., et al. (2014). Vacuolin-1 potently and reversibly inhibits autophagosome-lysosome fusion by activating RAB5A. Autophagy 10, 1895-1905. doi: 10.4161/auto.32200

Lystad, A. H., Carlsson, S. R., and Simonsen, A. (2019). Toward the function of mammalian ATG12-ATG5-ATG16L1 complex in autophagy and related processes. Autophagy 15, 1485-1486. doi: 10.1080/15548627.2019.1618100

Mao, K., and Klionsky, D. J. (2011). AMPK activates autophagy by phosphorylating ULK1. Circ. Res. 108, 787-788. doi: 10.1161/RES.0b013e3182194c29

Mele, L., Del Vecchio, V., Liccardo, D., Prisco, C., Schwerdtfeger, M., Robinson, N., et al. (2020). The role of autophagy in resistance to targeted therapies. Cancer Treat Rev. 88:102043. doi: 10.1016/j.ctrv.2020.102043

Moon, H. J., Park, S. Y., Lee, S. H., Kang, C. D., and Kim, S. H. (2019). Nonsteroidal anti-inflammatory drugs sensitize CD44-overexpressing cancer cells to Hsp90 inhibitor through autophagy activation. Oncol. Res. 27, 835-847. doi: 10.3727/ 096504019x15517850319579

Moosavi, M. S., and Elham, Y. (2020). Aquaporins 1, 3 and 5 in different tumors, their expression. prognosis value and role as new therapeutic targets. Pathol. Oncol. Res. 26, 615-625. doi: 10.1007/s12253-019-00646-9

Mrakovcic, M., and Fröhlich, L. F. (2018). p53-Mediated molecular control of autophagy in tumor cells. Biomolecules 8:14. doi: 10.3390/biom8020014 
Murugan, A. K. (2019). mTOR: Role in cancer, metastasis and drug resistance. Semin. Cancer Biol. 59, 92-111. doi: 10.1016/j.semcancer.2019.07.003

Nakayama, S., Karasawa, H., Suzuki, T., Yabuuchi, S., Takagi, K., Aizawa, T., et al. (2017). p62/sequestosome 1 in human colorectal carcinoma as a potent prognostic predictor associated with cell proliferation. Cancer Med. 6, 12641274. doi: $10.1002 / \mathrm{cam} 4.1093$

Nazim, U. M., Yin, H., and Park, S. Y. (2020). Downregulation of c-FLIP and upregulation of DR- 5 by cantharidin sensitizes TRAIL-mediated apoptosis in prostate cancer cells via autophagy flux. Int. J. Mol.Med. 46, 280-288. doi: 10.3892/ijmm.2020.4566

Nie, Y., Liang, X., Liu, S., Guo, F., Fang, N., and Zhou, F. (2020). WASF3 knockdown sensitizes gastric cancer cells to oxaliplatin by inhibiting ATG12mediated autophagy. Am. J. Med. Sci. 359, 287-295. doi: 10.1016/j.amjms.2020. 02.007

Nobili, A., Krashia, P., and D’Amelio, M. (2020). Cisd2: a promising new target in Alzheimer's disease(†). J. Pathol. 251, 113-116. doi: 10.1002/path.5436

Ouyang, L., Zhang, L., Fu, L., and Liu, B. (2017). A small-molecule activator induces ULK1-modulating autophagy-associated cell death in triple negative breast cancer. Autophagy 13, 777-778. doi: 10.1080/15548627.2017.1283470

Pan, X. K., Su, F., Xu, L. H., Yang, Z. S., Wang, D. W., Yang, L. J., et al. (2018). DJ-1 Alters Epirubicin-induced Apoptosis via modulating epirubicin activated autophagy in human gastric cancer cells. Curr. Med. Sci. 38, 1018-1024. doi: 10.1007/s11596-018-1978-y

Panda, P. K., Mukhopadhyay, S., Das, D. N., Sinha, N., Naik, P. P., and Bhutia, S. K. (2015). Mechanism of autophagic regulation in carcinogenesis and cancer therapeutics. Semin. Cell Dev. Biol. 39, 43-55. doi: 10.1016/j.semcdb.2015.02. 013

Parikh, C., Janakiraman, V., Wu, W. I., Foo, C. K., Kljavin, N. M., Chaudhuri, S., et al. (2012). Disruption of PH-kinase domain interactions leads to oncogenic activation of AKT in human cancers. Proc. Natl. Acad. Sci. U.S.A. 109, 1936819373. doi: $10.1073 /$ pnas. 1204384109

Pattingre, S., Bauvy, C., and Codogno, P. (2003). Amino acids interfere with the ERK1/2-dependent control of macroautophagy by controlling the activation of Raf-1 in human colon cancer HT-29 cells. J. Biol. Chem. 278, 16667-16674. doi: 10.1074/jbc.M210998200

Pei, G., Luo, M., Ni, X., Wu, J., Wang, S., Ma, Y., et al. (2018). Autophagy facilitates metadherin-induced chemotherapy resistance through the AMPK/ATG5 pathway in gastric cancer. Cell Physiol. Biochem. 46, 847-859. doi: 10.1159/ 000488742

Perez-Montoyo, H. (2020). Therapeutic potential of autophagy modulation in cholangiocarcinoma. Cells 9:614. doi: 10.3390/cells9030614

Petroni, G., Bagni, G., Iorio, J., Duranti, C., Lottini, T., Stefanini, M., et al. (2020). Clarithromycin inhibits autophagy in colorectal cancer by regulating the hERG1 potassium channel interaction with PI3K. Cell Death Dis. 11:161. doi: 10.1038/s41419-020-2349-8

Poursheikhani, A., Bahmanpour, Z., Razmara, E., Mashouri, L., Taheri, M., Morshedi Rad, D., et al. (2020). Non-coding RNAs underlying chemoresistance in gastric cancer. Cell Oncol. doi: 10.1007/s13402-020-00528-2 [Epub ahead of print].

Qi, Y., Lv, J., Liu, S., Sun, L., Wang, Y., Li, H., et al. (2019). TSPAN9 and EMILIN1 synergistically inhibit the migration and invasion of gastric cancer cells by increasing TSPAN9 expression. BMC Cancer 19:630. doi: 10.1186/s12885-019$5810-2$

Qi, Y., Qi, W., Liu, S., Sun, L., Ding, A., Yu, G., et al. (2020). TSPAN9 suppresses the chemosensitivity of gastric cancer to 5 -fluorouracil by promoting autophagy. Cancer Cell Int. 20:4. doi: 10.1186/s12935-019-1089-2

Qian, H. R., and Yang, Y. (2016). Functional role of autophagy in gastric cancer. Oncotarget 7, 17641-17651. doi: 10.18632/oncotarget.7508

Qin, J., Wang, W., and Zhang, R. (2017). Novel natural product therapeutics targeting both inflammation and cancer. Chin. J. Nat. Med. 15, 401-416. doi: 10.1016/S1875-5364(17)30062-6

Qin, J. J., Jin, H. Z., Huang, Y., Zhang, S. D., Shan, L., Voruganti, S., et al. (2013). Selective cytotoxicity, inhibition of cell cycle progression, and induction of apoptosis in human breast cancer cells by sesquiterpenoids from Inula lineariifolia Turcz. Eur. J. Med. Chem. 68, 473-481. doi: 10.1016/j.ejmech.2013. 07.018

Qin, J. J., Li, X., Hunt, C., Wang, W., Wang, H., and Zhang, R. (2018). Natural products targeting the $\mathrm{p} 53-\mathrm{MDM} 2$ pathway and mutant $\mathrm{p} 53$ : recent advances and implications in cancer medicine. Genes Dis. 5, 204-219. doi: 10.1016/j. gendis.2018.07.002

Qiu, L., Zhou, G., and Cao, S. (2020). Targeted inhibition of ULK1 enhances daunorubicin sensitivity in acute myeloid leukemia. Life Sci. 243:117234. doi: 10.1016/j.lfs.2019.117234

Ranek, M. J., Kokkonen-Simon, K. M., Chen, A., Dunkerly-Eyring, B. L., Vera, M. P., Oeing, C. U., et al. (2019). PKG1-modified TSC2 regulates mTORC1 activity to counter adverse cardiac stress. Nature 566, 264-269. doi: 10.1038/ s41586-019-0895-y

Ratti, M., Lampis, A., Ghidini, M., Salati, M., Mirchev, M. B., Valeri, N., et al. (2020). MicroRNAs (miRNAs) and long non-coding RNAs (lncRNAs) as new tools for cancer therapy: first steps from bench to bedside. Target Oncol. 15, 261-278. doi: 10.1007/s11523-020-00717-x

Reggiori, F., and Ungermann, C. (2017). Autophagosome maturation and fusion. J. Mol. Biol. 429, 486-496. doi: 10.1016/j.jmb.2017.01.002

Russi, S., Verma, H. K., Laurino, S., Mazzone, P., Storto, G., Nardelli, A., et al. (2019). Adapting and surviving: intra and extra-cellular remodeling in drugresistant gastric cancer cells. Int. J. Mol. Sci. 20:3736. doi: 10.3390/ijms20153736

Sanchez-Martin, P., Lahuerta, M., Viana, R., Knecht, E., and Sanz, P. (2020). Regulation of the autophagic PI3KC3 complex by laforin/malin E3-ubiquitin ligase, two proteins involved in Lafora disease. Biochim. Biophys. Acta Mol. Cell Res. 1867:118613. doi: 10.1016/j.bbamcr.2019.118613

Santos-Laso, A., Izquierdo-Sanchez, L., Rodrigues, P. M., Huang, B. Q., Azkargorta, M., Lapitz, A., et al. (2020). Proteostasis disturbances and endoplasmic reticulum stress contribute to polycystic liver disease: new therapeutic targets. Liver Int. 40, 1670-1685. doi: 10.1111/liv.14485

Sato, E., Ohta, S., Kawakami, K., Ikeda, M., Takahashi, T., Kobayashi, S., et al. (2019). Tetrandrine increases the sensitivity of human lung adenocarcinoma PC14 cells to Gefitinib by Lysosomal Inhibition. Anticancer Res. 39, 6585-6593. doi: 10.21873 /anticanres. 13874

Seetha, A., Devaraj, H., and Sudhandiran, G. (2020). Indomethacin and juglone inhibit inflammatory molecules to induce apoptosis in colon cancer cells. J. Biochem. Mol. Toxicol. 34:e22433. doi: 10.1002/jbt.22433

Shi, X., Yokom, A., Wang, C., Young, L., Youle, R., and Hurley, J. (2020). ULK complex organization in autophagy by a C-shaped FIP200 N-terminal domain dimer. J. Cell Biol. 219:e201911047. doi: 10.1083/jcb.201911047

Stanga, D., Zhao, Q., Milev, M., Saint-Dic, D., Jimenez-Mallebrera, C., and Sacher, M. (2019). TRAPPC11 functions in autophagy by recruiting ATG2BWIPI4/WDR45 to preautophagosomal membranes. Traffic 20, 325-345. doi: $10.1111 /$ tra. 12640

Suárez-Cabrera, C., de la Peña, B., González, L. L., Page, A., Martínez-Fernández, M., Casanova, M. L., et al. (2018). The Ras-related gene ERAS is involved in human and murine breast cancer. Sci. Rep. 8:13038. doi: 10.1038/s41598-01831326-4

Sun, H., Lesche, R., Li, D., Liliental, J., Zhang, H., Gao, J., et al. (1999). PTEN modulates cell cycle progression and cell survival by regulating phosphatidylinositol 3,4,5,-trisphosphate and Akt/protein kinase B signaling pathway. Proc. Natl. Acad. Sci. U.S.A. 96, 6199-6204. doi: 10.1073/pnas.96.11. 6199

Sun, Y., Jiang, Y., Huang, J., Chen, H., Liao, Y., and Yang, Z. (2017). CISD2 enhances the chemosensitivity of gastric cancer through the enhancement of 5-FU-induced apoptosis and the inhibition of autophagy by AKT/mTOR pathway. Cancer Med. 6, 2331-2346. doi: 10.1002/cam4.1169

Thrift, A. P., and El-Serag, H. B. (2019). Burden of gastric cancer. Clin. Gastroenterol. Hepatol. 18, 534-542. doi: 10.1016/j.cgh.2019.07.045

Tian, H., Wang, W., Meng, X., Wang, M., Tan, J., Jia, W., et al. (2019). ERas enhances resistance to cisplatin-induced apoptosis by suppressing autophagy in gastric cancer cell. Front. Cell Dev. Biol. 7:375. doi: 10.3389/fcell.2019.00375

Tian, L., Zhao, Z., Xie, L., and Zhu, J. (2018). MiR-361-5p suppresses chemoresistance of gastric cancer cells by targeting FOXM1 via the PI3K/Akt/mTOR pathway. Oncotarget 9, 4886-4896. doi: 10.18632/oncotarget. 23513

Tsai, C. Y., Lin, T. A., Huang, S. C., Hsu, J. T., Yeh, C. N., Chen, T. C., et al. (2020). Is adjuvant chemotherapy necessary for patients with deficient mismatch repair gastric cancer?-autophagy inhibition matches the mismatched. Oncologist 25 , e1021-e1030. doi: 10.1634/theoncologist.2019-0419

Vallecillo-Hernandez, J., Barrachina, M. D., Ortiz-Masia, D., Coll, S., Esplugues, J. V., Calatayud, S., et al. (2018). Indomethacin disrupts autophagic flux by 
inducing lysosomal dysfunction in gastric cancer cells and increases their sensitivity to cytotoxic drugs. Sci. Rep. 8:3593. doi: 10.1038/s41598-018-21 $455-1$

Wang, H., Jiao, H., Jiang, Z., and Chen, R. (2020). Propofol inhibits migration and induces apoptosis of pancreatic cancer PANC-1 cells through miR-34amediated E-cadherin and LOC285194 signals. Bioengineered 11, 510-521. doi: $10.1080 / 21655979.2020 .1754038$

Wei, F., Jiang, X., Gao, H. Y., and Gao, S. H. (2017). Liquiritin induces apoptosis and autophagy in cisplatin (DDP)-resistant gastric cancer cells in vitro and xenograft nude mice in vivo. Int. J. Oncol. 51, 1383-1394. doi: 10.3892/ijo.2017. 4134

Wu, J., Chen, S., Liu, H., Zhang, Z., Ni, Z., Chen, J., et al. (2018). Tunicamycin specifically aggravates ER stress and overcomes chemoresistance in multidrugresistant gastric cancer cells by inhibiting N-glycosylation. J. Exp. Clin. Cancer Res. 37:272. doi: 10.1186/s13046-018-0935-8

Xi, Z., Si, J., and Nan, J. (2019). LncRNA MALAT1 potentiates autophagyassociated cisplatin resistance by regulating the microRNA-30b/autophagyrelated gene 5 axis in gastric cancer. Int. J. Oncol. 54, 239-248. doi: 10.3892/ ijo.2018.4609

Xiao, F., Ouyang, B., Zou, J., Yang, Y., Yi, L., and Yan, H. (2020). Trim14 promotes autophagy and chemotherapy resistance of gastric cancer cells by regulating AMPK/mTOR pathway. Drug Dev. Res. 81, 544-550. doi: 10.1002/ddr.21650

Xin, L., Zhou, Q., Yuan, Y. W., Zhou, L. Q., Liu, L., Li, S. H., et al. (2019). METase/lncRNA HULC/FoxM1 reduced cisplatin resistance in gastric cancer by suppressing autophagy. J. Cancer Res. Clin. Oncol. 145, 2507-2517. doi: 10.1007/s00432-019-03015-w

Xu, J., Chen, Y., Yang, R., Zhou, T., Ke, W., Si, Y., et al. (2020). Cucurbitacin B inhibits gastric cancer progression by suppressing STAT3 activity. Arch. Biochem. Biophys. 684:108314. doi: 10.1016/j.abb.2020.10 8314

Xu, W., Shi, Q., Qian, X., Zhou, B., Xu, J., Zhu, L., et al. (2018a). Rab5a suppresses autophagy to promote drug resistance in cancer cells. Am. J. Transl. Res. 10, 1229-1236.

Xu, W., Wei, Q., Han, M., Zhou, B., Wang, H., Zhang, J., et al. (2018b). CCL2-SQSTM1 positive feedback loop suppresses autophagy to promote chemoresistance in gastric cancer. Int. J. Biol. Sci. 14, 1054-1066. doi: 10.7150/ ijbs. 25349

Xu, Z., Chen, L., Xiao, Z., Zhu, Y., Jiang, H., Jin, Y., et al. (2018). Potentiation of the anticancer effect of doxorubicinin drug-resistant gastric cancer cells by tanshinone IIA. Phytomedicine 51, 58-67. doi: 10.1016/j.phymed.2018.05.012

Yang, L. Q., Li, R. Y., Yang, X. Y., Cui, Q. F., Wang, F. Y., Lin, G. Q., et al. (2019). Co-administration of shexiang baoxin pill and chemotherapy drugs potentiated cancer therapy by vascular-promoting strategy. Front. Pharmacol. 10:565. doi: 10.3389/fphar.2019.00565

YiRen, H., YingCong, Y., Sunwu, Y., Keqin, L., Xiaochun, T., Senrui, C., et al. (2017). Long noncoding RNA MALAT1 regulates autophagy associated chemoresistance via miR-23b-3p sequestration in gastric cancer. Mol. Cancer 16:174. doi: 10.1186/s12943-017-0743-3

You, Q., Xu, J., Zhu, Z., Hu, Z., and Cai, Q. (2020). Phloretin flavonoid exhibits selective antiproliferative activity in doxorubicin-resistant gastric cancer cells by inducing autophagy, inhibiting cell migration and invasion, cell cycle arrest and targeting ERK1/2 MAP pathway. J. Buon. 25, 308-313.

Young, L. N., Goerdeler, F., and Hurley, J. H. (2019). Structural pathway for allosteric activation of the autophagic PI 3-kinase complex I. Proc. Natl. Acad. Sci. U.S.A. 116, 21508-21513. doi: 10.1073/pnas.191161 2116

Yu, Y., Hou, L., Song, H., Xu, P., Sun, Y., and Wu, K. (2017). Akt/AMPK/mTOR pathway was involved in the autophagy induced by vitamin $\mathrm{E}$ succinate in human gastric cancer SGC-7901 cells. Mol. Cell Biochem. 424, 173-183. doi: 10.1007/s11010-016-2853-4

Yuan, L., Xu, Z. Y., Ruan, S. M., Mo, S., Qin, J. J., and Cheng, X. D. (2020). Long non-coding RNAs towards precision medicine in gastric cancer: early diagnosis, treatment, and drug resistance. Mol. Cancer 19:96. doi: 10.1186/s12943-02001219-0

Yuan, L., Zhang, K., Zhou, M. M., Wasan, H. S., Tao, F. F., Yan, Q. Y., et al. (2019). Jiedu sangen decoction reverses epithelial-to-mesenchymal transition and inhibits invasion and metastasis of colon cancer via AKT/GSK-3 $\beta$ signaling pathway. J. Cancer 10, 6439-6456. doi: 10.7150/jca.32873

Zachari, M., and Ganley, I. G. (2017). The mammalian ULK1 complex and autophagy initiation. Essays Biochem. 61, 585-596. doi: 10.1042/ebc20170021

Zeng, X., Wang, H. Y., Bai, S. Y., Pu, K., Wang, Y. P., and Zhou, Y. N. (2020). The roles of microRNAs in multidrug-resistance mechanisms in gastric cancer. Curr. Mol. Med. doi: 10.2174/1566524020666200226124336 [Epub ahead of print].

Zhang, C., Wang, N., Tan, H. Y., Guo, W., Chen, F., Zhong, Z., et al. (2020). Direct inhibition of the TLR4/MyD88 pathway by geniposide suppresses HIF- $1 \alpha$-independent VEGF expression and angiogenesis in hepatocellular carcinoma. Br. J. Pharmacol. 177, 3240-3257. doi: 10.1111/bph.15046

Zhang, F., Chen, C., Hu, J., Su, R., Zhang, J., Han, Z., et al. (2019). Molecular mechanism of Helicobacter pylori-induced autophagy in gastric cancer. Oncol. Lett. 18, 6221-6227. doi: 10.3892/ol.2019.10976

Zhang, X., Wang, L., Ireland, S. C., Ahat, E., Li, J., Bekier, M. E., et al. (2019). GORASP2/GRASP55 collaborates with the PtdIns3K UVRAG complex to facilitate autophagosome-lysosome fusion. Autophagy 15, 1787-1800. doi: 10. $1080 / 15548627.2019 .1596480$

Zhang, X., Xie, K., Zhou, H., Wu, Y., Li, C., Liu, Y., et al. (2020). Role of noncoding RNAs and RNA modifiers in cancer therapy resistance. Mol. Cancer 19:47. doi: 10.1186/s12943-020-01171-z

Zhang, Y., Fan, Y., Huang, S., Wang, G., Han, R., Lei, F., et al. (2018). Thymoquinone inhibits the metastasis of renal cell cancer cells by inducing autophagy via AMPK/mTOR signaling pathway. Cancer Sci. 109, 3865-3873. doi: $10.1111 /$ cas. 13808

Zhang, Y. F., Li, C. S., Zhou, Y., and Lu, X. H. (2020). Propofol facilitates cisplatin sensitivity via lncRNA MALAT1/miR-30e/ATG5 axis through suppressing autophagy in gastric cancer. Life Sci. 244:117280. doi: 10.1016/j.lfs.2020.117280

Zhao, R., Zhang, X., Zhang, Y., Zhang, Y., Yang, Y., Sun, Y., et al. (2020). HOTTIP predicts poor survival in gastric cancer patients and contributes to cisplatin resistance by sponging miR-216a-5p. Front. Cell Dev. Biol. 8:348. doi: 10.3389/ fcell.2020.00348

Zhong, B., Li, Y., Liu, X., and Wang, D. (2018). Association of mast cell infiltration with gastric cancer progression. Oncol. Lett. 15, 755-764. doi: 10.3892/ol.2017. 7380

Zhou, H., Yuan, M., Yu, Q., Zhou, X., Min, W., and Gao, D. (2016). Autophagy regulation and its role in gastric cancer and colorectal cancer. Cancer Biomark 17, 1-10. doi: $10.3233 / \mathrm{cbm}-160613$

Zhou, S., Sun, Y., Zhao, K., Gao, Y., Cui, J., Qi, L., et al. (2020). miR-21/PTEN pathway mediates the cardioprotection of geniposide against oxidized lowdensity lipoprotein-induced endothelial injury via suppressing oxidative stress and inflammatory response. Int. J. Mol. Med. 45, 1305-1316. doi: 10.3892/ijmm. 2020.4520

Zhu, J. X., Qin, J. J., Jin, H. Z., and Zhang, W. D. (2013). Japonicones Q-T, four new dimeric sesquiterpene lactones from Inula japonica Thunb. Fitoterapia 84, 40-46. doi: 10.1016/j.fitote.2012.09.026

Conflict of Interest: The authors declare that the research was conducted in the absence of any commercial or financial relationships that could be construed as a potential conflict of interest.

Copyright $\odot 2020 X u, Y u a n$, Tang, Xu, Xu, Cheng and Qin. This is an open-access article distributed under the terms of the Creative Commons Attribution License $(C C B Y)$. The use, distribution or reproduction in other forums is permitted, provided the original author(s) and the copyright owner(s) are credited and that the original publication in this journal is cited, in accordance with accepted academic practice. No use, distribution or reproduction is permitted which does not comply with these terms. 